\title{
Measurement of inequality of opportunity: A normative approach
}

\author{
Kristof Bosmans $^{1}$ (D) . Z. Emel Öztürk ${ }^{2}$
}

Received: 15 September 2019 / Accepted: 27 September 2020 / Published online: 2 April 2021

(C) The Author(s) 2021

\begin{abstract}
We develop a normative approach to the measurement of inequality of opportunity. That is, we measure inequality of opportunity by the welfare gain obtained in moving from the actual income distribution to the optimal income distribution of the total available income. Our study brings together the main approaches in the literature: we axiomatically characterize social welfare functions, we obtain prominent allocation rules as their optima, and we derive familiar classes of inequality of opportunity measures. Our analysis captures moreover the key philosophical distinctions in the literature: ex post versus ex ante compensation, and liberal versus utilitarian reward.
\end{abstract}

Keywords Inequality of opportunity $\cdot$ Income inequality $\cdot$ Responsibility $\cdot$ Social welfare

\section{Introduction}

The ideal of equality of opportunity rests on two principles. The compensation principle condemns the inequalities that arise from individual circumstances (e.g., parental background). The reward principle, by contrast, condones or even justifies the inequalities due to the exercise of individual responsibility (e.g., work effort). Differentiating between circumstance and responsibility characteristics allows for a broad ethical range, from leftist positions that would put most individual characteristics in the circumstance basket, to rightist positions that would put most characteristics in the responsibility basket.

We develop a normative approach to the measurement of inequality of opportunity. In this approach, measures of inequality of opportunity are derived from social welfare

Kristof Bosmans

k.bosmans@maastrichtuniversity.nl

Z. Emel Öztürk

z.e.ozturk@tilburguniversity.edu

1 Department of Microeconomics and Public Economics, Maastricht University, Tongersestraat 53, 6211 LM Maastricht, The Netherlands

2 Department of Economics, Tilburg University, 5000 LE Tilburg, The Netherlands 
functions. ${ }^{1}$ We first characterize social welfare functions on the basis of axioms that express elementary ethical values. To derive inequality of opportunity measures, we define inequality of opportunity to be equal to the social welfare gain obtained in moving from the actual income distribution to the optimal income distribution of the total available income. The resulting inequality of opportunity measures inherit a normative foundation from the social welfare functions on which they are based.

The literature on equality of opportunity has grown rapidly since the pioneering contributions by Roemer (1993), Van de gaer (1993), Fleurbaey (1994) and Bossert (1995). ${ }^{2}$ Unfortunately, this literature lacks a unified framework within which contributions can be easily connected and compared. First, different contributions have adopted sharply distinct philosophical views on how to define the compensation and reward principles. As a result, there is no single agreed upon conceptualization of equality of opportunity, but rather several competing ones. Second, contributions have focused on a variety of criteria as the object of study. Some study social welfare functions, others study allocation rules and yet others study measures of inequality of opportunity. We argue that the normative approach is particularly useful to work towards a framework that encompasses the philosophical and methodological diversity of the literature.

First, the normative approach is an axiomatic approach, which makes it well suited to study the implications of different philosophical views. Our key axioms embody the different views in the literature on compensation and reward. The compensation principle comes in two versions. Ex post compensation (Roemer 1993; Fleurbaey 1994) says that the incomes of individuals exercising the same responsibility should be equalized, whereas ex ante compensation (Van de gaer 1993) says that the incomes of groups with different circumstances should be equalized. ${ }^{3}$ The reward principle also comes in two versions. Liberal reward (Fleurbaey 1994; Bossert and Fleurbaey 1996) says that income differences due to the exercise of responsibility should respect the market returns to responsibility, whereas utilitarian reward (Roemer 1993; Van de gaer 1993) says that all income differences due to responsibility are irrelevant. We employ axioms for the ex post and ex ante versions of compensation, for the liberal and utilitarian versions of reward, as well as for several variants of these. This allows the study of all these different philosophical outlooks within a single framework.

Second, the normative approach produces a natural connection between the different criteria that have been used to formalize the idea of equality of opportunity. The connection between the characterized social welfare functions and the derived inequality of opportunity measures is immediate. Moreover, we are naturally led to examine the allocation rules corresponding to the characterized social welfare functions because the optimal distribution of a given amount of income plays a crucial role in the normative approach. Our social welfare functions, allocation rules and inequality of opportunity measures all take forms that cover and generalize prominent classes in the literature. Our approach thus links together these different criteria in a unified framework.

\footnotetext{
${ }^{1}$ The normative approach was originally proposed in the simpler setting of income inequality measurement by Kolm (1969), Atkinson (1970) and Sen (1973).

${ }^{2}$ For surveys, see Fleurbaey (2008), Fleurbaey and Maniquet (2011), Fleurbaey and Schokkaert (2012), Pignataro (2012), Ramos and Van de gaer (2016), Roemer and Trannoy (2015, 2016) and Ferreira and Peragine (2016). The economic literature builds on earlier work in political philosophy: see Rawls (1971), Dworkin (1981a, b), Arneson (1989, 1990) and Cohen (1989).

${ }^{3}$ The distinction between the ex post and ex ante perspectives is due to Peragine (2004b) and Ooghe, Schokkaert and Van de gaer (2007). The latter link the ex ante perspective to the literature on equalizing opportunity sets (e.g., Kranich 1996, Ok 1997, Ok and Kranich 1998 and Weymark 2003).
} 
We proceed as follows. The next section introduces notation and some basic axioms that we always impose on the social welfare functions. Section 3 defines and discusses the axioms that capture the ex post and ex ante versions of compensation and the liberal and utilitarian versions of reward. Section 4 characterizes classes of social welfare functions using the basic axioms and different combinations of compensation and reward principles. Section 5 derives optimal distributions from the obtained social welfare functions. We link the induced allocation rules to those proposed in the literature. Section 6 explains the normative approach to the measurement of inequality of opportunity and derives the classes of inequality measures corresponding to the previously characterized classes of social welfare functions. The obtained inequality of opportunity measures are discussed in the light of the literature. Section 7 concludes.

\section{Preliminaries}

Each individual is characterized by his circumstance and responsibility characteristics. The set of all circumstance characteristics is $C=\{1,2, \ldots, c\}$ and the set of all responsibility characteristics is $R=\{1,2, \ldots, r\}$. For simplicity, we assume that each combination $(i, k)$ in $C \times R$ occurs exactly once. ${ }^{4}$ We refer to each $(i, k)$ as an individual.

We use a $c \times r$ real-valued matrix $X$ to represent an income distribution. The $i k$ th entry of $X$, denoted by $x_{i k}$, is the income of individual $(i, k)$. The $i$ th row of $X$ is denoted by $x_{i}$. and the $k$ th column is denoted by $x_{. k}$. We denote the average income in $X$ by $\bar{X}$. Similarly, we denote the average incomes in $x_{i}$. and $x_{\cdot k}$ by $\bar{x}_{i}$. and $\bar{x}_{{ }_{k}}$. We write $1_{c \times r}$ for the $c \times r$ matrix with 1 at each entry and $1_{r}$ for the $r$-dimensional vector with 1 at each entry.

As we discuss in Subsection 3.2, the liberal reward principle requires to respect the market returns to the exercise of responsibility. We therefore single out the distribution of laissez-faire market incomes. ${ }^{5}$ We denote this income distribution by $M$ in $\mathbb{R}^{c \times r}$ and refer to it simply as the market income distribution. ${ }^{6}$ We assume that $M$ is fixed. ${ }^{7}$

We use a social welfare function to compare income distributions. A social welfare function $W: \mathbb{R}^{c \times r} \rightarrow \mathbb{R}$ assigns to each income distribution $X$ in $\mathbb{R}^{c \times r}$ a real number $W(X)$. The function $W$ depends on $M$, but we suppress this dependency in the notation.

We impose axioms on the social welfare function to make concrete its normative properties. In the remainder of this section, we formulate three basic axioms. These axioms are standard in the literature on income inequality measurement. ${ }^{8}$ The next section discusses more substantive axioms representing the ideas of compensation and reward.

\footnotetext{
${ }^{4}$ The extension to the general case where some combinations occur more than once or do not occur at all is possible, but would require considerably heavier notation.

${ }^{5}$ These are the hypothetical incomes that individuals would obtain if government interference in the economy were minimal. What constitutes "minimal" interference depends on the particular strand of classical liberal or libertarian political thought (Mack and Gaus 2004).

${ }^{6}$ The literature commonly refers to market income as pre-tax income (e.g., Bossert 1995 and Bossert and Fleurbaey 1996). The few empirical studies that apply liberal inequality of opportunity measures do not attempt to estimate hypothetical laissez-faire income, but rather proxy it by pre-tax labor income (Devooght 2008; Almås et al. 2011).

${ }^{7}$ Requiring $M$ to be fixed is restrictive, as it prevents comparisons between countries or over time. However, the requirement is standard, if often implicit, in the literature (e.g., Bossert 1995, Bossert and Fleurbaey 1996 and Fleurbaey and Peragine 2013). In Section 7, we discuss the repercussions of allowing $M$ to vary.

${ }^{8}$ See Ebert (1988), Blackorby et al. (1999) and Cowell (2000) for surveys.
} 
Monotonicity says that increasing the income of an individual is socially desirable provided that no other individual's income decreases.

Monotonicity For all income distributions $X$ and $X^{\prime}$ in $\mathbb{R}^{c \times r}$, if $x_{i k} \geq x_{i k}^{\prime}$ for each individual $(i, k)$ in $C \times R$ and $x_{j l}>x_{j l}^{\prime}$ for some individual $(j, l)$ in $C \times R$, then $W(X)>W\left(X^{\prime}\right)$.

Continuity ensures that social welfare comparisons are not overly sensitive to small changes in the income distributions.

Continuity The function $W$ is continuous.

Translation invariance demands that the social welfare ranking of two income distributions does not change if the same amount is added to each income in both income distributions. $^{9}$

Translation invariance For all income distributions $X$ and $X^{\prime}$ in $\mathbb{R}^{c \times r}$ and for each real number $\lambda$, we have $W(X) \geq W\left(X^{\prime}\right)$ if and only if $W\left(X+\lambda 1_{c \times r}\right) \geq W\left(X^{\prime}+\lambda 1_{c \times r}\right)$.

Translation invariance ensures that the inequality of opportunity measures we derive later are absolute. That is, adding the same amount to each income does not change the level of inequality of opportunity.

\section{Compensation and reward}

\subsection{Compensation axioms}

The compensation principle says that income inequalities due to differences in circumstances ought to be eliminated. There are two versions of compensation, ex post compensation and ex ante compensation. To understand the terminology, imagine that circumstance characteristics are determined prior to responsibility characteristics. Ex ante compensation is defined in terms of the income possibilities of circumstance groups before responsibility characteristics are determined, whereas ex post compensation is defined in terms of the actual incomes that arise after responsibility characteristics are also determined.

Ex post compensation comprises two components, a Pigou-Dalton transfer principle and a symmetry principle. Together, these components express the idea that individuals who exercise the same responsibility should be treated equally. Ex post Pigou-Dalton requires that an income transfer that widens the income gap between two individuals in the same responsibility group reduces social welfare.

Ex post Pigou-Dalton For all income distributions $X$ and $X^{\prime}$ in $\mathbb{R}^{c \times r}$, if there exist two individuals $(i, k)$ and $(j, k)$ in $C \times R$ such that $x_{i k} \geq x_{j k}$ and a positive real number $\delta$ such

\footnotetext{
${ }^{9}$ In Section 7, we discuss how our results change if translation invariance is replaced by scale invariance. Scale invariance says that the social welfare ranking of two income distributions should not change if we multiply each income with the same factor in both income distributions.
} 
that $x_{i k}^{\prime}=x_{i k}+\delta$ and $x_{j k}^{\prime}=x_{j k}-\delta$ with $X$ and $X^{\prime}$ coinciding everywhere else, then $W(X)>W\left(X^{\prime}\right)$.

Ex post symmetry demands that switching the incomes of two individuals in the same responsibility group does not change social welfare.

Ex post symmetry For all income distributions $X$ and $X^{\prime}$ in $\mathbb{R}^{c \times r}$, if there exist two individuals $(i, k)$ and $(j, k)$ in $C \times R$ such that $x_{i k}=x_{j k}^{\prime}$ and $x_{j k}=x_{i k}^{\prime}$ with $X$ and $X^{\prime}$ coinciding everywhere else, then $W(X)=W\left(X^{\prime}\right)$.

We refer to the combination of ex post Pigou-Dalton and ex post symmetry as ex post compensation. Note that both ex post Pigou-Dalton and ex post symmetry were already proposed by Peragine (2004b).

Ex post compensation Both ex post Pigou-Dalton and ex post symmetry hold.

Next, we define the ex ante version of compensation. To understand ex ante compensation, interpret row $i$ of an income distribution as the (income) opportunities of an individual with circumstance characteristic $i$. Ex ante compensation says that differences in circumstances do not justify differences in these opportunities. The axiom consists of, again, a Pigou-Dalton transfer and a symmetry component.

Ex ante Pigou-Dalton requires that increasing the gap between opportunities decreases social welfare. Assume that the minimum income in circumstance group $i$ is greater than the maximum income in circumstance group $j$. We can then conclude that group $i$ is unambiguously better off than group $j$. Now, imagine a transfer from each individual in $j$ to each individual in $i$. Ex ante Pigou-Dalton requires that such a transfer reduces social welfare. This Pigou-Dalton axiom is based on an axiom of Fleurbaey and Peragine (2013). ${ }^{10}$

Ex ante Pigou-Dalton For all income distributions $X$ and $X^{\prime}$ in $\mathbb{R}^{c \times r}$, if there exist two circumstance groups $i$ and $j$ in $C$ such that $\min _{k \in R} x_{i k} \geq \max _{k \in R} x_{j k}$ and a positive real number $\delta$ such that $x_{i}^{\prime} .=x_{i} .+\delta 1_{r}$ and $x_{j}^{\prime} .=x_{j} .-\delta 1_{r}$ with $X$ and $X^{\prime}$ coinciding everywhere else, then $W(X)>W\left(X^{\prime}\right)$.

Ex ante symmetry requires that switching two rows of an income distribution-one row again unambiguously better off than the other as defined above-does not change social welfare. Note that a similar symmetry axiom has appeared in Ooghe et al. (2007).

Ex ante symmetry For all income distributions $X$ and $X^{\prime}$ in $\mathbb{R}^{c \times r}$, if there exist two circumstance groups $i$ and $j$ in $C$ such that $\min _{k \in R} x_{i k} \geq \max _{k \in R} x_{j k}$ and $x_{i}$. $=x_{j}^{\prime}$. and $x_{j} .=x_{i}^{\prime}$. with $X$ and $X^{\prime}$ coinciding everywhere else, then $W(X)=W\left(X^{\prime}\right)$.

We refer to the combination of ex ante Pigou-Dalton and ex ante symmetry as ex ante compensation.

Ex ante compensation Both ex ante Pigou-Dalton and ex ante symmetry hold.

\footnotetext{
${ }^{10}$ It is based on their ex ante Pigou-Dalton compensation, the weakest among the ex ante compensation axioms they propose.
} 
Obviously, ex post Pigou-Dalton implies ex ante Pigou-Dalton and ex post symmetry implies ex ante symmetry. By consequence, ex post compensation implies ex ante compensation.

Ex ante Pigou-Dalton is weaker than two alternative axioms that have appeared in the literature. The key difference between the three axioms lies in how circumstance groups are ranked. The axiom of Fleurbaey and Peragine (2013) regards circumstance group $i$ as better off than circumstance group $j$ if, for each responsibility category, the income in $i$ is greater than the income in $j .{ }^{11}$ The axiom of Peragine (2004a) ranks circumstance groups on the basis of average income. ${ }^{12}$ As we discuss in Section 4.2, strengthening ex ante compensation in the direction of either of these alternative axioms does not alter the corresponding characterization result. Hence, ex ante compensation has the advantage of parsimony.

\subsection{Reward axioms}

The reward principle complements the compensation principle. Whereas compensation aims to neutralize differences in circumstances, reward tells us how to respect differences in responsibility. The literature considers two versions of reward, liberal reward and utilitarian reward.

Liberal reward states that differences in the market incomes of individuals in the same circumstance group should be respected. A useful restatement of this idea is that each individual in the same circumstance class should receive the same subsidy, where a subsidy is defined as the actual income minus the market income.

Liberal reward consists of two components, a Pigou-Dalton transfer principle and a symmetry principle. Consider two individuals in the same circumstance group $i$. The subsidies received by $(i, k)$ and $(i, l)$ in income distribution $X$ are $x_{i k}-m_{i k}$ and $x_{i l}-m_{i l}$. Assume that the subsidy received by $(i, k)$ is greater than the subsidy received by $(i, l)$. Liberal PigouDalton requires that transferring income from $(i, l)$ to $(i, k)$ reduces social welfare, as such an income transfer further widens the gap between the subsidies received by the two individuals. Note that Ramos and Van de gaer (2016) proposed a similar axiom, inspired by a stronger axiom of Fleurbaey and Peragine (2013).

Liberal Pigou-Dalton For all income distributions $X$ and $X^{\prime}$ in $\mathbb{R}^{c \times r}$, if there exist two individuals $(i, k)$ and $(i, l)$ in $C \times R$ such that $x_{i k}-m_{i k} \geq x_{i l}-m_{i l}$ and a positive real number $\delta$ such that $x_{i k}^{\prime}=x_{i k}+\delta$ and $x_{i l}^{\prime}=x_{i l}-\delta$ with $X$ and $X^{\prime}$ coinciding everywhere else, then $W(X)>W\left(X^{\prime}\right)$.

We illustrate the axiom with an example. Imagine a society with one circumstance group and three responsibility groups. Consider the income distributions $X=(9,9,15)$ and $X^{\prime}=$ $(10,8,15)$. The market income distribution is $M=(7,9,14)$. The distributions of subsidies in $X$ and $X^{\prime}$ are $X-M=(2,0,1)$ and $X^{\prime}-M=(3,-1,1)$. The gap between the subsidies

\footnotetext{
${ }^{11}$ The axiom we refer to is their strong ex ante compensation, the strongest among the ex ante compensation axioms they propose.

${ }^{12}$ Peragine's (2004a) axiom shares with ex ante Pigou-Dalton that it considers a set of transfers affecting all individuals in the two circumstance groups. The axiom of Fleurbaey and Peragine (2013), by contrast, considers a single transfer (that need not preserve total income) that affects only one individual in each of the two circumstance groups. Note that, contrary to ex ante compensation, both of these alternative axioms clash with ex post compensation (see Fleurbaey and Peragine 2013 and Ramos and Van de gaer 2016).
} 
received by the first two individuals is smaller in $X$ than in $X^{\prime}$. Thus, liberal Pigou-Dalton says that $X$ is better than $X^{\prime} .^{13}$

Liberal symmetry demands that switching the subsidies of two individuals in the same circumstance group leaves social welfare unchanged. Note that such a switch does not alter the total income of the circumstance group.

Liberal symmetry For all income distributions $X$ and $X^{\prime}$ in $\mathbb{R}^{c \times r}$, if there exist two individuals $(i, k)$ and $(i, l)$ in $C \times R$ such that $x_{i k}-m_{i k}=x_{i l}^{\prime}-m_{i l}$ and $x_{i l}-m_{i l}=x_{i k}^{\prime}-m_{i k}$ with $X$ and $X^{\prime}$ coinciding everywhere else, then $W(X)=W\left(X^{\prime}\right)$.

To illustrate the axiom, let $X^{\prime \prime}=(7,11,15)$ and $M=(7,9,14)$. Liberal symmetry says that $X^{\prime \prime}$ and $X=(9,9,15)$ are equally good since $X^{\prime \prime}-M=(0,2,1)$ is obtained from $X-M=(2,0,1)$ by switching the subsidies received by the first two individuals.

We refer to the combination of liberal Pigou-Dalton and liberal symmetry as liberal reward.

Liberal reward Both liberal Pigou-Dalton and liberal symmetry hold.

Liberal reward says that, for an individual with circumstances $i$, the move from responsibility $k$ to $l$ should be rewarded as it is rewarded by the market, that is, by an income change of $m_{i l}-m_{i k}$. We stress that the same axiom can capture alternative reward principles by letting $M$ be, instead of the market income distribution, an income distribution featuring alternative ideal income differences, e.g., based on an independent concept of desert. ${ }^{14}$

Next, we define utilitarian reward. Utilitarian reward takes the agnostic view that equality of opportunity should be silent on how to reward differences in responsibility. Accordingly, utilitarian reward requires the social welfare function to be neutral with respect to transfers within a circumstance group. There is no need to separately define Pigou-Dalton transfer and symmetry components for utilitarian reward since the axiom as stated includes both ideas. The axiom was introduced by Peragine (2004b).

Utilitarian reward For all income distributions $X$ and $X^{\prime}$ in $\mathbb{R}^{c \times r}$, if there exist two individuals $(i, k)$ and $(i, l)$ in $C \times R$ and a positive real number $\delta$ such that $x_{i k}^{\prime}=x_{i k}+\delta$ and $x_{i l}^{\prime}=x_{i l}-\delta$ with $X$ and $X^{\prime}$ coinciding everywhere else, then $W(X)=W\left(X^{\prime}\right)$.

\section{Social welfare functions}

\subsection{Compensation and liberal reward}

We first focus on the combination of ex post compensation and liberal reward. As the following example shows, these two axioms clash. Assume that there are two circumstance groups and two responsibility groups. Consider

$$
X=\left[\begin{array}{ll}
3 & 5 \\
3 & 5
\end{array}\right], \quad X^{\prime}=\left[\begin{array}{ll}
1 & 7 \\
5 & 3
\end{array}\right] \text { and } \quad M=\left[\begin{array}{cc}
4 & 10 \\
2 & 0
\end{array}\right]
$$

\footnotetext{
${ }^{13}$ Repeated use of liberal Pigou-Dalton yields the income distribution $(8,10,15)$. In this income distribution, each individual receives a subsidy of 1 .

${ }^{14}$ See Fleurbaey (2008, pp. 221-223) and Roemer (2012, pp. 178-179) for discussions of alternative reward principles.
} 
We have

$$
X-M=\left[\begin{array}{rr}
-1 & -5 \\
1 & 5
\end{array}\right] \quad \text { and } \quad X^{\prime}-M=\left[\begin{array}{rr}
-3 & -3 \\
3 & 3
\end{array}\right] .
$$

Ex post Pigou-Dalton implies $W(X)>W\left(X^{\prime}\right)$, whereas liberal Pigou-Dalton implies $W\left(X^{\prime}\right)>W(X)$.

Ex post compensation and liberal reward can be combined only if market income can be written as an additively separable function of circumstance and responsibility characteristics. ${ }^{15}$ We say that market incomes are additively separable if $m_{i k}-m_{i l}=m_{j k}-m_{j l}$ for all circumstance groups $i$ and $j$ and all responsibility groups $k$ and $l .{ }^{16}$ That is, a given change in responsibility causes the same change in market income, irrespective of the circumstance group.

Proposition 1 A social welfare function that satisfies ex post Pigou-Dalton and liberal Pigou-Dalton exists only if market incomes are additively separable.

In the example above, market incomes are not additively separable. Consider the change from the first to the second responsibility category. Market income changes by 6 in the first circumstance group, but by -2 in the second. This makes it impossible to simultaneously equalize income in each responsibility group (as favored by ex post compensation) and respect market income differences in each circumstance group (as favored by liberal reward). For instance, the income distribution

$$
X^{\prime \prime}=\left[\begin{array}{ll}
1 & 7 \\
1 & 7
\end{array}\right]
$$

meets the former requirement, but only meets the latter requirement for the first circumstance group. Clearly, having equality in both columns while respecting market incomes in both rows is possible only if market incomes are additively separable. ${ }^{17}$

We will in two ways deal with the incompatibility between ex post Pigou-Dalton and liberal Pigou-Dalton. First, we combine the axioms under the restriction of additively separable market incomes (Theorem 1). Second, we consider compromise versions of liberal reward and ex post compensation (Theorems 2 and 3 ).

Theorem 1 restricts market incomes to be additively separable, and characterizes social welfare functions that satisfy ex post compensation and liberal reward in addition to the three basic axioms monotonicity, continuity and translation invariance. As we will see in Section 5, the social welfare functions in Theorem 1 extend the "natural" allocation rule. We denote the set of $c r$-dimensional real valued vectors by $\mathbb{R}^{c r}$.

Theorem 1 Let market incomes in $M$ be additively separable. A social welfare function $W$ satisfies monotonicity, continuity, translation invariance, ex post compensation and liberal

\footnotetext{
${ }^{15}$ This is a well known result in the setting of allocation rules. See, for example, Bossert (1995) and Bossert and Fleurbaey (1996).

${ }^{16}$ Bossert (1995) and Bossert and Fleurbaey (1996) considered a function $f$ that assigns to each individual $(i, k)$ a market income $f(i, k)$. The market income function $f$ is additively separable if there exist functions $g$ and $h$ such that, for each $(i, k)$ in $C \times R$, we have $f(i, k)=g(i)+h(k)$. This is equivalent to the condition that, for all $i$ and $j$ in $C$ and $k$ and $l$ in $R$, we have $f(i, k)-f(i, l)=f(j, k)-f(j, l)$. This clearly corresponds to our definition of additive separability of market incomes.

${ }^{17}$ Consider an alternative market income distribution for which a change from the first to the second responsibility category corresponds to a change in market income of 6 in both circumstance groups. Then market incomes are additively separable. In this case, for a total income amount of 16 to be distributed, the income distribution $X^{\prime \prime}$ is the optimum that corresponds to the "natural" allocation rule. See Section 5.
} 
reward if and only if there exists a strictly increasing, continuous, translatable ${ }^{18}$ and strictly Schur-concave ${ }^{19}$ function $f: \mathbb{R}^{c r} \rightarrow \mathbb{R}$ such that, for each $X$ in $\mathbb{R}^{c \times r}$,

$$
W(X)=f\left(x_{11}-m_{11}+\bar{m}_{1 .}, \ldots, x_{i k}-m_{i k}+\bar{m}_{i .}, \ldots, x_{c r}-m_{c r}+\bar{m}_{c} .\right) .
$$

We explain why the social welfare function in Theorem 1 satisfies ex post compensation and liberal reward. (The intuition for Theorems 2 and 3 is similar.) First, for any two individuals in the same responsibility group, the same number is subtracted from their incomes. Indeed, additive separability of market incomes implies $m_{i k}-\bar{m}_{i} .=m_{j k}-\bar{m}_{j}$. for all circumstance groups $i$ and $j$ and each responsibility group $k$. Strict Schur-concavity of $f$ then ensures that ex post compensation is satisfied. Second, for any two individuals in the same circumstance group, what goes into $f$ is their subsidies plus a uniform constant. Again, strict Schur-concavity of $f$ guarantees that liberal reward is satisfied.

Next, we drop the restriction that market incomes are additively separable, and consider compromise versions of the liberal reward and ex post compensation axioms. These compromise versions are inspired by the axioms equal transfer for reference circumstance and equal income for reference responsibility of Bossert and Fleurbaey (1996).

We start with the compromise version of liberal reward. The idea is to use for each circumstance group the market incomes of a predetermined circumstance group $\hat{c}$ instead of the group's actual market incomes. Liberal reward is then guaranteed only with respect to the reference circumstance group $\hat{c}$. The compromise version of liberal Pigou-Dalton is as follows.

Liberal Pigou-Dalton for $\hat{c}$ Let $\hat{c}$ be a circumstance characteristic in $C$. For all income distributions $X$ and $X^{\prime}$ in $\mathbb{R}^{c \times r}$, if there exist two individuals $(i, k)$ and $(i, l)$ in $C \times R$ such that $x_{i k}-m_{\hat{c} k} \geq x_{i l}-m_{\hat{c} l}$ and a positive real number $\delta$ such that $x_{i k}^{\prime}=x_{i k}+\delta$ and $x_{i l}^{\prime}=x_{i l}-\delta$ with $X$ and $X^{\prime}$ coinciding everywhere else, then $W(X)>W\left(X^{\prime}\right)$.

The corresponding version of the liberal symmetry axiom is as follows.

Liberal symmetry for $\hat{c}$ Let $\hat{c}$ be a circumstance characteristic in $C$. For all income distributions $X$ and $X^{\prime}$ in $\mathbb{R}^{c \times r}$, if there exist two individuals $(i, k)$ and $(i, l)$ in $C \times R$ such that $x_{i k}-m_{\hat{c} k}=x_{i l}^{\prime}-m_{\hat{c} l}$ and $x_{i l}-m_{\hat{c} l}=x_{i k}^{\prime}-m_{\hat{c} k}$ with $X$ and $X^{\prime}$ coinciding everywhere else, then $W(X)=W\left(X^{\prime}\right)$.

Liberal reward for $\hat{c}$ combines liberal Pigou-Dalton for $\hat{c}$ and liberal symmetry for $\hat{c}$. Note that if market incomes are additively separable, then liberal reward for $\hat{c}$ is equivalent to liberal reward. If market incomes are not additively separable, then the two axioms clash.

Liberal reward for $\hat{c}$ Both liberal Pigou-Dalton for $\hat{c}$ and liberal symmetry for $\hat{c}$ hold.

\footnotetext{
${ }^{18}$ A function $f: \mathbb{R}^{n} \rightarrow \mathbb{R}$ is translatable if we have $f(x) \geq f(y)$ if and only if $f\left(x+\delta 1_{n}\right) \geq f\left(y+\delta 1_{n}\right)$ for all $x$ and $y$ in $\mathbb{R}^{n}$ and each real number $\delta$.

${ }^{19} \mathrm{~A}$ bistochastic matrix is a nonnegative square matrix of which each row sums to 1 and each column sums to 1 . A permutation matrix is a bistochastic matrix of which each component is either 0 or 1 . A function $f: \mathbb{R}^{n} \rightarrow \mathbb{R}$ is Schur-concave if $f(B x) \geq f(x)$ for each $x$ in $\mathbb{R}^{n}$ and each $n \times n$ bistochastic matrix $B$. If, in addition, $f(B x)>f(x)$ whenever $B$ is not a permutation matrix, then $f$ is strictly Schur-concave. Note that (strict) Schur-concavity of $f$ implies symmetry of $f$.
} 
Theorem 2 characterizes the social welfare functions that satisfy ex post compensation and liberal reward for $\hat{c}$ in addition to the three basic axioms. The result follows easily from Theorem $1^{20}$ and we state it without proof. In Section 5 we will see that the social welfare functions in the theorem extend the egalitarian-equivalent allocation rule.

Theorem 2 A social welfare function $W$ satisfies monotonicity, continuity, translation invariance, ex post compensation and liberal reward for $\hat{c}$ if and only if there exists a strictly increasing, continuous, translatable and strictly Schur-concave function $f: \mathbb{R}^{c r} \rightarrow \mathbb{R}$ such that, for each $X$ in $\mathbb{R}^{c \times r}$,

$$
W(X)=f\left(x_{11}-m_{\hat{c} 1}, \ldots, x_{i k}-m_{\hat{c} k}, \ldots, x_{c r}-m_{\hat{c} r}\right) .
$$

Next, we turn to the compromise version of ex post compensation. This version guarantees ex post Pigou-Dalton only with respect to a chosen responsibility group $\hat{r}$. The compromise version of ex post Pigou-Dalton is as follows.

Ex post Pigou-Dalton for $\hat{r}$ Let $\hat{r}$ be a responsibility characteristic in $R$. For all income distributions $X$ and $X^{\prime}$ in $\mathbb{R}^{c \times r}$, if there exist two individuals $(i, k)$ and $(j, k)$ in $C \times R$ such that $x_{i k}-m_{i k}+m_{i \hat{r}} \geq x_{j k}-m_{j k}+m_{j \hat{r}}$ and a positive real number $\delta$ such that $x_{i k}^{\prime}=x_{i k}+\delta$ and $x_{j k}^{\prime}=x_{j k}-\delta$ with $X$ and $X^{\prime}$ coinciding everywhere else, then $W(X)>W\left(X^{\prime}\right)$.

The corresponding version of ex post symmetry is as follows.

Ex post symmetry for $\hat{r}$ Let $\hat{r}$ be a responsibility characteristic in $R$. For all income distributions $X$ and $X^{\prime}$ in $\mathbb{R}^{c \times r}$, if there exist two individuals $(i, k)$ and $(j, k)$ in $C \times R$ such that $x_{i k}-m_{i k}+m_{i \hat{r}}=x_{j k}^{\prime}-m_{j k}+m_{j \hat{r}}$ and $x_{j k}-m_{j k}+m_{j \hat{r}}=x_{i k}^{\prime}-m_{i k}+m_{i \hat{r}}$ with $X$ and $X^{\prime}$ coinciding everywhere else, then $W(X)=W\left(X^{\prime}\right)$.

Ex post compensation for $\hat{r}$ combines ex post Pigou-Dalton for $\hat{r}$ and ex post symmetry for $\hat{r}$. Note that if market incomes are additively separable, then ex post compensation for $\hat{r}$ coincides with ex post compensation. If not, then the two axioms clash.

Ex post compensation for $\hat{r}$ Both ex post Pigou-Dalton for $\hat{r}$ and ex post symmetry for $\hat{r}$ hold.

Theorem 3 characterizes the social welfare functions that satisfy ex post compensation for $\hat{r}$ and liberal reward in addition to the three basic axioms. The proof is similar to that of Theorem 1 and is therefore omitted. Section 5 will show that the social welfare functions in the theorem extend the conditional equality allocation rule.

Theorem 3 A social welfare function W satisfies monotonicity, continuity, translation invariance, ex post compensation for $\hat{r}$ and liberal reward if and only if there exists a strictly increasing, continuous, translatable and strictly Schur-concave function $f: \mathbb{R}^{c r} \rightarrow \mathbb{R}$ such that, for each $X$ in $\mathbb{R}^{c \times r}$,

$$
W(X)=f\left(x_{11}-m_{11}+m_{1 \hat{r}}, \ldots, x_{i k}-m_{i k}+m_{i \hat{r}}, \ldots, x_{c r}-m_{c r}+m_{c \hat{r}}\right) .
$$

\footnotetext{
${ }^{20}$ Replace $M$ by the income distribution of which each row equals $m_{\hat{c}}$. and apply Theorem 1 .
} 
We now move on to the combination of ex ante compensation and liberal reward. As the following example shows, ex ante symmetry and liberal Pigou-Dalton clash. Consider the income distributions

$$
X=\left[\begin{array}{cc}
10 & 14 \\
1 & 3
\end{array}\right], X^{\prime}=\left[\begin{array}{cc}
1 & 3 \\
10 & 14
\end{array}\right], X^{\prime \prime}=\left[\begin{array}{cc}
0 & 4 \\
11 & 13
\end{array}\right] \text { and } X^{\prime \prime \prime}=\left[\begin{array}{cc}
11 & 13 \\
0 & 4
\end{array}\right]
$$

and the market income distribution

$$
M=\left[\begin{array}{cc}
10 & 14 \\
1 & 3
\end{array}\right]
$$

We have $W(X)=W\left(X^{\prime}\right)$ by ex ante symmetry, $W\left(X^{\prime}\right)<W\left(X^{\prime \prime}\right)$ by liberal Pigou-Dalton and $W\left(X^{\prime \prime}\right)=W\left(X^{\prime \prime \prime}\right)$ by ex ante symmetry. Hence, $W(X)<W\left(X^{\prime \prime \prime}\right)$. However, we have $W\left(X^{\prime \prime \prime}\right)<W(X)$ by liberal Pigou-Dalton. ${ }^{21}$

We again obtain that a necessary condition to avoid the clash is that market incomes are additively separable.

Proposition 2 A social welfare function $W$ that satisfies ex ante symmetry and liberal Pigou-Dalton exists only if market incomes are additively separable.

For the case of ex ante compensation, we will not explore domain restrictions and compromise axioms. We suffice instead by remarking that all the social welfare functions in Theorems 1 and 2 satisfy ex ante compensation, as the latter is implied by ex post compensation.

\subsection{Compensation and utilitarian reward}

We begin with the combination of ex post compensation and utilitarian reward. The following example shows that the two axioms clash. ${ }^{22}$ Consider the income distributions

$$
X=\left[\begin{array}{ll}
8 & 7 \\
6 & 9
\end{array}\right] \text { and } X^{\prime}=\left[\begin{array}{ll}
7 & 8 \\
7 & 8
\end{array}\right] \text {. }
$$

Ex post Pigou-Dalton implies $W\left(X^{\prime}\right)>W(X)$, whereas utilitarian reward implies $W(X)=$ $W\left(X^{\prime}\right)$.

We introduce uniform utilitarian reward, a weakening of utilitarian reward, and combine it with ex post compensation. ${ }^{23}$ Uniform utilitarian reward consists of a Pigou-Dalton transfer and a symmetry component. Uniform utilitarian Pigou-Dalton says that transferring the same amount $\delta$ from each individual in a responsibility group to each individual in another responsibility group should not alter social welfare.

\footnotetext{
${ }^{21}$ Suppose that a total income amount of 28 , the same amount as in the example, has to be distributed. The income distribution with $(5,9)$ as the first row and $(6,8)$ as the second seems the obvious candidate for an optimum that captures both ex ante compensation and liberal reward. Indeed, this income distribution equalizes the average incomes of the circumstance groups and respects the market income differences within each circumstance group. This income distribution is the optimum corresponding to the observable average conditional equality allocation rule introduced by Bossert et al. (1999). This allocation rule is characterized by axioms that are direct translations of ex ante compensation and liberal reward to the setting of allocation rules (Bossert et al. 1999; Bosmans and Öztürk 2021). See Bosmans et al. (2020) for inequality of opportunity measures consistent with this allocation rule.

${ }^{22}$ This example is essentially the same as that used by Ramos and Van de gaer (2016) to establish a related incompatibility.

${ }^{23}$ Since utilitarian reward does not take market incomes into account, a restriction on the domain of market income distributions is not an option in this case.
} 
Uniform utilitarian Pigou-Dalton For all income distributions $X$ and $X^{\prime}$ in $\mathbb{R}^{c \times r}$, if there exist two responsibility groups $k$ and $l$ in $R$ and a positive real number $\delta$ such that $x_{\cdot k}^{\prime}=$ $x_{\cdot k}+\delta 1_{c}$ and $x_{\cdot l}^{\prime}=x_{\cdot l}-\delta 1_{c}$ with $X$ and $X^{\prime}$ coinciding everywhere else, then $W(X)=$ $W\left(X^{\prime}\right)$.

Uniform utilitarian symmetry demands that switching two columns of an income distribution does not change social welfare.

Uniform utilitarian symmetry For all income distributions $X$ and $X^{\prime}$ in $\mathbb{R}^{c \times r}$, if there exist two responsibility groups $k$ and $l$ in $R$ such that $x_{\cdot k}=x_{\cdot l}^{\prime}$ and $x_{\cdot l}=x_{\cdot k}^{\prime}$ with $X$ and $X^{\prime}$ coinciding everywhere else, then $W(X)=W\left(X^{\prime}\right)$.

We refer to the combination of uniform utilitarian Pigou-Dalton and uniform utilitarian symmetry as uniform utilitarian reward.

Uniform utilitarian reward Both uniform utilitarian Pigou-Dalton and uniform utilitarian symmetry hold.

We impose an additional axiom that puts structure on social welfare comparisons. The axiom requires that the social welfare function first aggregates the incomes of each responsibility group and second aggregates the obtained values across circumstance groups. Because this order of aggregation requires knowledge of individuals' responsibility characteristics, we refer to the axiom as ex post aggregation. Note that the axiom was proposed by Peragine (2004b).

Ex post aggregation There exist a function $\phi: \mathbb{R}^{r} \rightarrow \mathbb{R}$ and functions $\gamma_{1}, \ldots, \gamma_{r}$ : $\mathbb{R}^{c} \rightarrow \mathbb{R}$ such that, for each income distribution $X$ in $\mathbb{R}^{c \times r}$, we have $W(X)=$ $\phi\left(\gamma_{1}\left(x_{\cdot 1}\right), \ldots, \gamma_{r}\left(x_{\cdot r}\right)\right)$.

Theorem 4 characterizes social welfare functions that satisfy ex post compensation and uniform utilitarian reward in addition to ex post aggregation and the three basic axioms monotonicity, continuity and translation invariance.

Theorem 4 A social welfare function $W$ satisfies monotonicity, continuity, translation invariance, ex post aggregation, ex post compensation and uniform utilitarian reward if and only if there exist a strictly increasing and continuous function $F: \mathbb{R} \rightarrow \mathbb{R}$ and a strictly increasing, continuous, unit-translatable ${ }^{24}$ and strictly Schur-concave function $f: \mathbb{R}^{c} \rightarrow \mathbb{R}$ such that, for each $X$ in $\mathbb{R}^{c \times r}$,

$$
W(X)=F\left(\frac{1}{r} \sum_{k \in R} f\left(x_{\cdot k}\right)\right) .
$$

The social welfare functions in the theorem first aggregate the incomes in each responsibility group using the function $f$. Strict Schur-concavity of $f$ ensures that ex post compensation is satisfied. The obtained values are then aggregated by averaging, which ensures satisfaction of uniform utilitarian reward.

\footnotetext{
${ }^{24} \mathrm{~A}$ function $f: \mathbb{R}^{n} \rightarrow \mathbb{R}$ is unit-translatable if $f\left(x+\delta 1_{n}\right)=f(x)+\delta$ for each $x$ in $\mathbb{R}^{n}$ and each real number $\delta$.
} 
We now turn to the combination of ex ante compensation and utilitarian reward. Theorem 5 characterizes social welfare functions that satisfy ex ante compensation and utilitarian reward in addition to the three basic axioms.

Theorem 5 A social welfare function $W$ satisfies monotonicity, continuity, translation invariance, ex ante compensation and utilitarian reward if and only if there exists a strictly increasing, continuous, translatable and strictly Schur-concave function $f: \mathbb{R}^{c} \rightarrow \mathbb{R}$ such that, for each $X$ in $\mathbb{R}^{c \times r}$,

$$
W(X)=f\left(\frac{1}{r} \sum_{k \in R} x_{1 k}, \frac{1}{r} \sum_{k \in R} x_{2 k}, \ldots, \frac{1}{r} \sum_{k \in R} x_{c k}\right) .
$$

The social welfare functions in the theorem first average the incomes of each circumstance group, thus ensuring satisfaction of utilitarian reward. These averages are then aggregated using the strictly Schur-concave function $f$, which ensures satisfaction of ex ante compensation.

Following our discussion in Section 3.1, consider two strengthenings of ex ante compensation. The first strengthening, inspired by Fleurbaey and Peragine (2013), regards circumstance group $i$ as better off than circumstance group $j$ if, for each responsibility category, the income in $i$ is greater than the income in $j$. The Pigou-Dalton component requires that a transfer from any member of group $j$ to any member of group $i$ reduces social welfare, and the symmetry component requires that switching the income vectors of groups $i$ and $j$ does not change social welfare. The second strengthening, inspired by Peragine (2004b), is defined in the same way, except that it compares circumstance groups on the basis of average income. It is easy to show that each of these two stronger axioms is implied by the combination of ex ante compensation and utilitarian reward. By consequence, the social welfare functions in Theorem 5 also satisfy these two strengthenings.

We end this subsection by connecting Theorems 4 and 5 with the previous literature. The social welfare function underlying Roemer's (1993) mean-of-mins allocation rule, $\frac{1}{r} \sum_{k \in R} \min _{i \in C} x_{i k}$, is not a member of the class in Theorem 4, but can be approached arbitrarily closely by choosing $f$ sufficiently concave. Likewise, the social welfare function underlying Van de gaer's (1993) min-of-means allocation rule, $\min _{i \in C} \frac{1}{r} \sum_{k \in R} x_{i k}$, while not a member of the class in Theorem 5 , can be approached arbitrarily closely by choosing $f$ sufficiently concave. Ooghe et al. (2007) characterize lexicographic versions of the meanof-mins and min-of-means social welfare orderings, as well as extensions thereof related to the classes in Theorems 4 and 5. Peragine (2004b), whose interest lies in unanimous rankings by classes of social welfare functions, likewise studies classes related to those in Theorems 4 and $5 .^{25}$

\section{Allocation rules}

A crucial step in the normative approach to inequality of opportunity measurement is to determine how a given amount of total income should be distributed in order to maximize social welfare. We now present the optimal income distributions for the social welfare

\footnotetext{
${ }^{25}$ In Section 7, we discuss unanimous rankings by our classes of social welfare functions and inequality of opportunity measures.
} 
functions characterized in the previous section. As we will see, these optima correspond to established allocation rules.

Proposition 3 presents the optimal distributions for the classes of social welfare functions in Theorems 1 to 5. In each case, the whole class settles on the same optimal distributions. The proof of the proposition is straightforward and is therefore omitted. We say that $X^{*}$ is an optimal distribution of the total income amount $\operatorname{cr} \mu$ if $\bar{X}^{*}=\mu$ and $W\left(X^{*}\right) \geq W(X)$ for each $X$ in $\left\{X \in \mathbb{R}^{c \times r}: \bar{X}=\mu\right\}$.

Proposition 3 Let $W$ be a social welfare function that satisfies monotonicity, continuity and translation invariance.

(i) Let $W$ satisfy, in addition, ex post compensation and liberal reward, and let market incomes in $M$ be additively separable (Theorem 1). The unique optimal distribution $X^{*}$ in $\mathbb{R}^{c \times r}$ of the total income amount cr $\mu$ is such that

$$
x_{i k}^{*}=m_{i k}-\bar{m}_{i .}+\mu \quad \text { for each }(i, k) \text { in } C \times R .
$$

(ii) Let $W$ satisfy, in addition, ex post compensation and liberal reward for $\hat{c}$ (Theorem 2). The unique optimal distribution $X^{*}$ in $\mathbb{R}^{c \times r}$ of the total income amount cr $\mu$ is such that

$$
x_{i k}^{*}=m_{\hat{c} k}-\bar{m}_{\hat{c} .}+\mu \quad \text { for each }(i, k) \text { in } C \times R .
$$

(iii) Let $W$ satisfy, in addition, ex post compensation for $\hat{r}$ and liberal reward (Theorem 3). The unique optimal distribution $X^{*}$ in $\mathbb{R}^{c \times r}$ of the total income amount cr $\mu$ is such that

$$
x_{i k}^{*}=m_{i k}-m_{i \hat{r}}+\bar{m}_{\cdot \hat{r}}-\bar{M}+\mu \quad \text { for each }(i, k) \text { in } C \times R .
$$

(iv) Let $W$ satisfy, in addition, ex post aggregation, ex post compensation and uniform utilitarian reward (Theorem 4). Each optimal distribution $X^{*}$ in $\mathbb{R}^{c \times r}$ of the total income amount $\mathrm{cr} \mu$ is such that

$$
x_{i k}^{*}=x_{j k}^{*} \quad \text { for all } i \text { and } j \text { in } C \text { and each } k \text { in } R .
$$

(v) Let $W$ satisfy, in addition, ex ante compensation and utilitarian reward (Theorem 5). Each optimal distribution $X^{*}$ in $\mathbb{R}^{c \times r}$ of the total income amount $\mathrm{cr} \mu$ is such that

$$
\bar{x}_{i}^{*}=\bar{x}_{j}^{*} . \quad \text { for all } i \text { and } j \text { in } C .
$$

Assume that $\mu=\bar{M}$. The optima in Proposition 3(i), 3(ii) to 3(iii) coincide, respectively, with the "natural", egalitarian-equivalent and conditional equality allocation rules introduced by Bossert (1995) and Bossert and Fleurbaey (1996). ${ }^{26}$ The "natural" allocation rule applies only if market incomes in $M$ are additively separable. Additive separability implies that there exist a real number $a_{i}$ for each circumstance group $i$ in $C$ and a real number $b_{k}$ for each responsibility group $k$ in $R$ such that $m_{i k}=a_{i}+b_{k}$. The "natural" allocation rule assigns to each individual $(i, k)$ the income $b_{k}+\bar{a}$, i.e., the part of his market income determined by responsibility plus the average of the part determined by circumstances. The allocation rule in Proposition 3(i) indeed coincides with the "natural" allocation rule since $m_{i k}=a_{i}+b_{k}, \bar{m}_{i} .=a_{i}+\bar{b}$ and $\mu=\bar{M}=\bar{a}+\bar{b}$. The egalitarian-equivalent allocation rule, obtained in Proposition 3(ii), assigns to each individual $(i, k)$ the income $m_{\hat{c} k}-\bar{m}_{\hat{c}}$. $+\bar{M}$, i.e., the market income he would have received if his circumstance were $\hat{c}$ plus a uniform

\footnotetext{
${ }^{26}$ If $\mu \neq \bar{M}$, then each individual receives what he would have received under these allocation rules plus the difference $\mu-\bar{M}$.
} 
amount. The conditional equality allocation rule, obtained in Proposition 3(iii), assigns to each individual $(i, k)$ the income $m_{i k}-m_{i \hat{r}}+\bar{m}_{. \hat{r}}$, i.e., the average market income of the responsibility group $\hat{r}$ plus the amount by which the individual's market income deviates from the market income he would have had were $k$ equal to $\hat{r}$.

The optima in Proposition 3(iv) and 3(v) coincide with the allocation rules proposed by Roemer (1993) and Van de gaer (1993). Indeed, the income distributions in Proposition 3(iv) maximize Roemer's mean-of-mins, $\frac{1}{r} \sum_{k \in R} \min _{i \in C} x_{i k}$, and those in 3(v) maximize Van de gaer's min-of-means, $\min _{i \in C} \frac{1}{r} \sum_{k \in R} x_{i k}$.

\section{Inequality of opportunity measures}

The normative approach to inequality measurement identifies inequality with the welfare loss incurred by having the actual rather than the optimal distribution of the total available income. First, we review the procedure proposed by Kolm (1969), Atkinson (1970) and Sen (1973) to derive measures of income inequality. Next, we extend this procedure to our setting. As we will see later, the Kolm-Atkinson-Sen (KAS) income inequality measure constitutes the basic building block of our measures of inequality of opportunity. Note that we consider absolute measures of income inequality and inequality of opportunity, that is, adding the same amount to each income leaves the level of inequality unaltered.

Consider the income inequality setting in which all individuals are identical. Let $x$ in $\mathbb{R}^{n}$ be an income distribution for $n$ individuals, and let $w: \mathbb{R}^{n} \rightarrow \mathbb{R}$ be a strictly increasing, continuous, translatable and strictly Schur-concave social welfare function. The equally distributed equivalent income $\xi(x)$ associated with $w$ is the income that, if received by each individual, would yield the same welfare level as $x$. Formally, $\xi(x)$ is the real number such that $w\left(\xi(x) 1_{n}\right)=w(x)$. The function $J: \mathbb{R}^{n} \rightarrow \mathbb{R}$ is said to be the KAS inequality measure associated with $w$ if

$$
J(x)=\bar{x}-\xi(x) \quad \text { for each } x \text { in } \mathbb{R}^{n} .
$$

The KAS measure has an intuitive interpretation. For each $x$ in $\mathbb{R}^{n}, J(x)$ is the per capita income that could be destroyed if incomes are equalized while maintaining the same level of welfare. It is a measure of waste due to inequality.

Next, we extend the above procedure to our setting. The difference with the income inequality setting is that the equal distribution is not necessarily optimal. Let $X$ in $\mathbb{R}^{c \times r}$ be an income distribution, and let $W: \mathbb{R}^{c \times r} \rightarrow \mathbb{R}$ be a strictly increasing and continuous social welfare function. The optimally distributed equivalent average income $\Xi(X)$ is the average income that, if distributed optimally among the individuals, would yield the same welfare level as $X$. Formally, $\Xi(X)=\bar{Y}$ with $Y$ such that $W(Y)=W(X)$ and $W(Y) \geq W(Z)$ for each $Z$ in $\mathbb{R}^{c \times r}$ for which $\bar{Z}=\bar{Y}$. The function $I: \mathbb{R}^{c \times r} \rightarrow \mathbb{R}$ is said to be the inequality of opportunity measure associated with $W$ if

$$
I(X)=\bar{X}-\Xi(X) \quad \text { for each } X \text { in } \mathbb{R}^{c \times r} .
$$

For each $X$ in $\mathbb{R}^{c \times r}, I(X)$ is the per capita income that could be destroyed if income is optimally distributed while maintaining the same level of welfare.

Proposition 4 presents the inequality of opportunity measures corresponding to the social welfare functions described in the five theorems in Section 4.

Proposition 4 Let $W$ be a social welfare function that satisfies monotonicity, continuity and translation invariance. 
(i) Let $W$ satisfy, in addition, ex post compensation and liberal reward, and let market incomes in $M$ be additively separable (Theorem 1). For each income distribution $X$ in $\mathbb{R}^{c \times r}$, we have

$$
I(X)=J\left(x_{11}-m_{11}+\bar{m}_{1 .}, \ldots, x_{i k}-m_{i k}+\bar{m}_{i .}, \ldots, x_{c r}-m_{c r}+\bar{m}_{c .}\right),
$$

where $J: \mathbb{R}^{c r} \rightarrow \mathbb{R}$ is the KAS inequality measure associated with $f$ in Eq. 1.

(ii) Let $W$ satisfy, in addition, ex post compensation and liberal reward for $\hat{c}$ (Theorem 2). For each income distribution $X$ in $\mathbb{R}^{c \times r}$, we have

$$
I(X)=J\left(x_{11}-m_{\hat{c} 1}, \ldots, x_{i k}-m_{\hat{c} k}, \ldots, x_{c r}-m_{\hat{c} r}\right),
$$

where $J: \mathbb{R}^{c r} \rightarrow \mathbb{R}$ is the KAS inequality measure associated with $f$ in Eq. 2.

(iii) Let $W$ satisfy, in addition, ex post compensation for $\hat{r}$ and liberal reward (Theorem 3). For each income distribution $X$ in $\mathbb{R}^{c \times r}$, we have

$$
I(X)=J\left(x_{11}-m_{11}+m_{1 \hat{r}}, \ldots, x_{i k}-m_{i k}+m_{i \hat{r}}, \ldots, x_{c r}-m_{c r}+m_{c \hat{r}}\right),
$$

where $J: \mathbb{R}^{c r} \rightarrow \mathbb{R}$ is the KAS inequality measure associated with $f$ in Eq. 3.

(iv) Let $W$ satisfy, in addition, ex post aggregation, ex post compensation and uniform utilitarian reward (Theorem 4). For each income distribution $X$ in $\mathbb{R}^{c \times r}$, we have

$$
I(X)=\frac{1}{r} \sum_{k \in R} J\left(x_{\cdot k}\right),
$$

where $J: \mathbb{R}^{c} \rightarrow \mathbb{R}$ is the KAS inequality measure associated with $f$ in Eq. 4 .

(v) Let $W$ satisfy, in addition, ex ante compensation and utilitarian reward (Theorem 5). For each income distribution $X$ in $\mathbb{R}^{c \times r}$, we have

$$
I(X)=J\left(\frac{1}{r} \sum_{k \in R} x_{1 k}, \frac{1}{r} \sum_{k \in R} x_{2 k}, \ldots, \frac{1}{r} \sum_{k \in R} x_{c k}\right),
$$

where $J: \mathbb{R}^{c} \rightarrow \mathbb{R}$ is the KAS inequality measure associated with $f$ in Eq. 5 .

Proposition 4 reveals that inequality of opportunity measurement reduces to the application of an income inequality measure to an appropriately adjusted income distribution. Our approach singles out the absolute KAS inequality measure as the income inequality measure to be employed. ${ }^{27}$ This use of income inequality measures as a basic building block is ubiquitous in the equality of opportunity literature, though not surprisingly without the restriction to absolute KAS inequality measures. ${ }^{28}$ We now discuss the five parts of Proposition 4 in connection with the previous literature.

The measures in Proposition 4(i) to 4(iii) apply an income inequality measure to a distribution of adjusted incomes where the adjustment term is determined by the market income distribution. The measures in Proposition 4(ii) and 4(iii) correspond, respectively, to the fairness gap and direct unfairness measures proposed by Fleurbaey and Schokkaert (2009).

Alternatively, the measures in Proposition 4(i) to 4(iii) can be written as measures of distance between the vector of actual incomes and the vector of optimal incomes. Indeed, these

\footnotetext{
${ }^{27}$ We provide an example of how the choice of $f$ fixes the choice of $J$. Assume that $f$ is the constant inequality aversion social welfare function, i.e., for each $x$ in $\mathbb{R}^{n}, f(x)=\ln \left[(1 / n) \sum_{i=1}^{n}-e^{-\alpha x_{i}}\right]$ with $\alpha>$ 0 . Note that this function satisfies all the properties imposed on $f$ in Theorems 1 to 5 . The equally distributed equivalent income is $\xi(x)=-(1 / \alpha) \ln \left[(1 / n) \sum_{i=1}^{n} e^{-\alpha x_{i}}\right]$. The KAS inequality measure associated with $f$ is $J(x)=(1 / \alpha) \ln \left[(1 / n) \sum_{i=1}^{n} e^{-\alpha\left(x_{i}-\bar{x}\right)}\right]$, which is Kolm's (1976) income inequality measure.

${ }^{28}$ In Section 7, we discuss variations of our approach that would warrant the use of a wider class of income inequality measures.
} 
measures are equivalent to the application of $J$ to the vector $\left(x_{11}-x_{11}^{*}, \ldots, x_{c r}-x_{c r}^{*}\right)$, where $x_{i k}^{*}$ is the optimal income as given in Proposition 3(i) to 3(iii). ${ }^{29}$ The measures proposed by Devooght (2008) and Almås et al. (2011) use this idea of distance between the actual and the optimal. Devooght (2008) uses the optimum corresponding to the egalitarian-equivalent rule as in Proposition 4(ii), but employs Cowell's (1985) measure of distributional change as a measure of distance. Almås et al. (2011) use the optimum corresponding to the so-called generalized proportionality rule (Cappelen and Tungodden 2017) and adopt the relative Gini index as a measure of distance. ${ }^{30}$ The advantage of our approach is that both the optimum and the distance measure follow from the axioms imposed on the social welfare function.

The measure in Proposition 4(iv) measures inequality of opportunity by the sum of the inequality levels of the responsibility groups. Aaberge et al. (2011) propose a measure in this form with $J$ a rank-dependent inequality measure. The measure in Proposition 4(iv) can be interpreted as measuring the inequality within responsibility groups while disregarding the (unproblematic) inequality between responsibility groups. This interpretation has been exploited by Checchi and Peragine (2010), who propose, among other things, the within responsibility group component of the mean logarithmic deviation as a measure of inequality of opportunity. See also Checchi et al. (2015), Brunori (2017) and Ramos and Van de gaer (2021).

The measure in Proposition 4(v) applies an inequality measure to the vector of the average incomes of the circumstance groups. This measure is dual to the measure in Proposition 4(iv) in the sense that it measures the inequality between circumstance groups while disregarding the (unproblematic) inequality within circumstance groups (Checchi and Peragine 2010). From the ex ante perspective, the average income of circumstance group $i$ represents the value of the opportunities of an individual with circumstance characteristic $i$. In this interpretation, the measure in Proposition 4(v) directly gauges the inequality between the opportunities of the circumstance groups. Measures of this ilk have been used extensively (with various choices of $J$ ) in empirical analysis. See, for example, Bourguignon et al. (2007), Cogneau and Mesplé-Somps (2008), Ferreira and Gignoux (2011), Ferreira et al. (2011), Belhaj Hassine (2012), Singh (2012), Marrero and Rodríguez (2013), Piraino (2015), Peichl and Ungerer (2016), Hufe et al. (2017), Álvarez and Menéndez (2018), Assaad et al. (2018) and Brunori et al. (2019). ${ }^{31}$

Finally, we discuss two measures that are not members of the class in Proposition 4(v), but are closely related. Lefranc et al. (2008) propose to measure the value of the opportunities of a circumstance group, not by the average income, but by the equally distributed equivalent income associated with the Gini social welfare function. They apply the relative Gini index to these equally distributed equivalent incomes. ${ }^{32}$ Unlike the measures in Proposition 4(v), their measure does not satisfy utilitarian reward, but rather a reward principle that disfavors inequality within circumstance groups (see also Ramos and Van de gaer

\footnotetext{
${ }^{29}$ Consider, for example, the measure in Proposition 4(iii). Because $J$ is absolute, we have $J\left(x_{11}-m_{11}+\right.$ $\left.m_{1 \hat{r}}, \ldots, x_{c r}-m_{c r}+m_{c \hat{r}}\right)=J\left(x_{11}-x_{11}^{*}, \ldots, x_{c r}-x_{c r}^{*}\right)$, where $x_{i k}^{*}=m_{i k}-m_{i \hat{r}}-\left(\bar{M}-\bar{m}_{\cdot \hat{r}}\right)+\bar{X}$.

${ }^{30}$ See Mahler and Ramos (2019) for an application of these methods to a variety of well-being measures.

${ }^{31}$ The previously mentioned studies by Aaberge et al. (2011), Checchi et al. (2015), Brunori (2017) and Ramos and Van de gaer (2021) also apply measures of this type.

${ }^{32}$ See Andreoli et al. (2019) for a generalization that allows for a broader set of social welfare functions and income inequality measures. Their setting moreover incorporates the element of luck: a combination of circumstance and responsibility characteristics determines a lottery over income, and luck decides which income realizes. Their interest lies in equality of lotteries rather than equality of realized incomes. See also Lefranc et al. (2009).
} 
2016). Second, the utilitarian ex post partial ordering introduced by Fleurbaey et al. (2017) suggests a measure that can be defined as follows. First, order the incomes in each column of the income distribution from poorest to richest. Next, apply an income inequality measure to the vector of the average incomes of the rows. Interestingly, even though the measure is of the form of the measures in Proposition 4(v), it is consistent with ex post compensation.

\section{Concluding remarks}

We conclude with four remarks. First, we consider rankings of income distributions agreed upon by all members of a class of inequality of opportunity measures. It is easy to obtain empirically tractable criteria for unanimous rankings by the classes in Proposition 4(i), 4(ii), 4(iii) and 4(v). Consider first the class in Proposition 4(i). Let $\tilde{x}=\left(x_{11}-x_{11}^{*}, \ldots, x_{c r}-x_{c r}^{*}\right)$, where $x_{i k}^{*}$ is the optimal income as given in Proposition 3(i). We have $I(X) \leq I\left(X^{\prime}\right)$ for each inequality of opportunity measure $I$ in Proposition 4(i) if and only if $\tilde{x}$ absolute Lorenz dominates $\tilde{x}^{\prime}{ }^{33}$ The same equivalence result holds for the classes in Proposition 4(ii) and 4(iii). Consider next the class in Proposition 4(v). Again, it is easy to establish that we have $I(X) \leq I\left(X^{\prime}\right)$ for each inequality of opportunity measure $I$ in Proposition 4(v) if and only if $\left(\bar{x}_{1 .}, \bar{x}_{2 .}, \ldots, \bar{x}_{c}\right.$. $)$ absolute Lorenz dominates $\left.\left(\bar{x}_{1 .}^{\prime}, \bar{x}_{2 .}^{\prime}, \ldots, \bar{x}_{c .}^{\prime}\right)\right)^{34}$

Second, we discuss how to obtain relative instead of absolute measures of inequality of opportunity. This requires replacing translation invariance by scale invariance, according to which the social welfare ranking of two income distributions does not change if each income in both income distributions is multiplied by the same factor. This leads to a straightforward change in Theorem 5: the function $f$ in Eq. 5 becomes homogeneous instead of translatable. For the other theorems, the required changes are less straightforward. Scale invariance clashes with liberal reward and liberal reward for $\hat{c} .{ }^{35}$ Hence, to obtain relative variants of Theorems 1, 2 and 3, we would need to replace liberal reward and liberal reward for $\hat{c}$ with versions that require to respect relative rather than absolute differences in market incomes within circumstance groups. Uniform utilitarian reward and scale invariance do not clash. ${ }^{36}$ However, uniform utilitarian reward is not in the spirit of relative inequality as the transfers are in equal absolute amounts. Therefore, to obtain a relative variant of Theorem 4, uniform utilitarian reward would have to be replaced by a version in which the transfers are proportional to income. We leave these variations for future research.

\footnotetext{
${ }^{33}$ For each $x$ in $\mathbb{R}^{n}$, let $\hat{x}$ be a rearrangement of $x$ such that $\hat{x}_{1} \leq \hat{x}_{2} \leq \cdots \leq \hat{x}_{n}$. For $x$ and $x^{\prime}$ in $\mathbb{R}^{n}$, we say that $x$ generalized Lorenz dominates $x^{\prime}$ if $\sum_{i=1}^{k} \hat{x}_{i} \geq \sum_{i=1}^{k} \hat{x}_{i}^{\prime}$ for each $k=1,2, \ldots, n$. We say that $x$ absolute Lorenz dominates $x^{\prime}$ if $\left(x-\bar{x} 1_{n}\right)$ generalized Lorenz dominates $\left(x^{\prime}-\bar{x}^{\prime} 1_{n}\right)$. See Shorrocks $(1983)$ and Moyes $(1987,1999)$.

${ }^{34}$ Analogous equivalence results are easy to obtain for the social welfare functions in Theorems 1, 2, 3 and 5. For example, we have $W(X) \geq W\left(X^{\prime}\right)$ for each social welfare function $W$ in Theorem 1 if and only if $\tilde{x}$ generalized Lorenz dominates $\tilde{x}^{\prime}$. Peragine (2004b) provides equivalence results for classes related to those in Theorems 4 and 5. Peragine's (2004b) class differs crucially from our class in Theorem 4 in that it uses a different aggregator function for each responsibility group. Having the same aggregator function for each responsibility group makes it difficult to obtain equivalence results for Theorem 4 and Proposition 4(iv).

${ }^{35}$ Assume there is one circumstance group and two responsibility groups. Let $M=(0,4), X=(2,6)$ and $X^{\prime}=(3,5)$. Liberal Pigou-Dalton implies $W(X)>W\left(X^{\prime}\right)$. Next, let $Y=4 X=(8,24)$ and $Y^{\prime}=4 X^{\prime}=$ $(12,20)$. Scale invariance implies $W(Y)>W\left(Y^{\prime}\right)$, but liberal Pigou-Dalton implies $W(Y)<W\left(Y^{\prime}\right)$.

${ }^{36}$ For each $x$ in $\mathbb{R}^{c}$, let $\hat{x}$ be a rearrangement of $x$ such that $\hat{x}_{1} \leq \hat{x}_{2} \leq \cdots \leq \hat{x}_{c}$. Consider a social welfare function in the form of Eq. 4, where, for each $x$ in $\mathbb{R}^{c}, f(x)=\sum_{i=1}^{c} a_{i} \hat{x}_{i}$ with $a_{1}>a_{2}>\cdots>a_{c}$ and $\sum_{i=1}^{c} a_{i}=1$. This social welfare function satisfies all the axioms in Theorem 4 and, in addition, scale invariance.
} 
Third, we outline the direct approach to inequality of opportunity measurement as an alternative to the normative approach. In the direct approach, axioms are directly imposed on measures of inequality of opportunity. In our axioms, statements on social welfare would have to be replaced by reverse statements on inequality of opportunity (e.g., $W(X)>$ $W\left(X^{\prime}\right)$ becomes $\left.I(X)<I\left(X^{\prime}\right)\right)$. The only substantial changes needed would be to omit monotonicity and to strengthen translation invariance so that it requires inequality of opportunity to remain unaltered if the same amount is added to each income. The advantage of such a direct approach is that it allows a wider class of income inequality measuresnot only the normative ones-to serve as building blocks for the measures of inequality of opportunity. The disadvantage is that the connection between inequality of opportunity measures and social welfare functions would be lost.

Fourth, we discuss a more challenging extension, relevant only for the classes that satisfy some version of liberal reward, namely, to allow for a variable market income distribution. This would be needed for comparisons between countries or over time. This extension suggests a stronger version of monotonicity that requires the pair $(X, M)$ to yield a higher level of social welfare than the pair $\left(X^{\prime}, M^{\prime}\right)$ if $x_{i k} \geq x_{i k}^{\prime}$ for each individual $(i, k)$ and $x_{j l}>x_{j l}^{\prime}$ for some individual $(j, l)$, regardless of the change from $M$ to $M^{\prime}$. However, as we have shown in a simpler setting (Bosmans and Öztürk 2015), this stronger monotonicity axiom clashes with liberal reward and continuity. We leave the question of how to incorporate variable market income distributions in the measurement of inequality of opportunity for future research.

\section{Appendix}

Proof of Proposition 1 Let $W$ be a social welfare function that satisfies ex post PigouDalton and liberal Pigou-Dalton.

Assume to the contrary that market incomes are not additively separable. That is, there exist $i$ and $j$ in $C$ and $k$ and $l$ in $R$ such that $m_{i k}-m_{i l} \neq m_{j k}-m_{j l}$. Let $X$ be an income distribution such that $x_{i k}=x_{j k}=\left(m_{i k}+m_{j k}\right) / 2$ and $x_{i l}=x_{j l}=\left(m_{i l}+m_{j l}\right) / 2$. Let $X^{\prime}$ be an income distribution such that $x_{i k}^{\prime}+x_{i l}^{\prime}=x_{i k}+x_{i l}, x_{i k}^{\prime}-x_{i l}^{\prime}=m_{i k}-m_{i l}$, $x_{j k}^{\prime}+x_{j l}^{\prime}=x_{j k}+x_{j l}$ and $x_{j k}^{\prime}-x_{j l}^{\prime}=m_{j k}-m_{j l}$ with $X^{\prime}$ and $X$ coinciding everywhere else. Ex post Pigou-Dalton implies $W(X)>W\left(X^{\prime}\right)$, whereas liberal Pigou-Dalton implies $W\left(X^{\prime}\right)>W(X)$. We have a contradiction.

The following two lemmas are used throughout the proofs. A progressive transfer is a transfer of income from a richer to a poorer individual such that the one that starts out with less money does not end up with more than the other. We say that a function is Pigou-Dalton consistent if its value increases as a result of a progressive transfer. See Olkin and Marshall (1979, pp. 10-12) for the first lemma and Dasgupta et al. (1973, p. 183) for the second lemma.

Lemma 1 For all vectors $a$ and $b$ in $\mathbb{R}^{n}$, $a$ is obtained from $b$ by a finite sequence of progressive transfers and permutations if and only if $a=b B$ for some $n \times n$ bistochastic matrix $B$.

Lemma 2 Each symmetric and Pigou-Dalton consistent function $f: \mathbb{R}^{n} \rightarrow \mathbb{R}$ is Schurconcave. 
Proof of Theorem 1 It is easy to verify that the specified social welfare function satisfies the axioms in the case of additively separable market incomes. We focus on the reverse implication.

Let $W$ be a social welfare function that satisfies the axioms.

By monotonicity and continuity, there exists a strictly increasing and continuous function $\hat{f}: \mathbb{R}^{c r} \rightarrow \mathbb{R}$ such that, for each $X$ in $\mathbb{R}^{c \times r}$, we have $W(X)=\hat{f}\left(\left(x_{i k}\right)_{(i, k) \in C \times R}\right)$. Translation invariance implies that, for all $x$ and $x^{\prime}$ in $\mathbb{R}^{c r}$ and each real number $\lambda$, we have $\hat{f}(x) \geq \hat{f}\left(x^{\prime}\right)$ if and only if $\hat{f}\left(x+\lambda 1_{c r}\right) \geq \hat{f}\left(x^{\prime}+\lambda 1_{c r}\right)$, i.e., $\hat{f}$ is a translatable function. Let $f$ be the function $f: \mathbb{R}^{c r} \rightarrow \mathbb{R}$ such that, for each vector $\left(x_{i k}\right)_{(i, k) \in C \times R}$, we have $f\left(\left(x_{i k}-m_{i k}+\bar{m}_{i} .\right)_{(i, k) \in C \times R}\right)=\hat{f}\left(\left(x_{i k}\right)_{(i, k) \in C \times R}\right)$. It follows that, for each $X$ in $\mathbb{R}^{c \times r}$, we have $W(X)=f\left(x_{11}-m_{11}+\bar{m}_{1 .}, \ldots, x_{i k}-m_{i k}+\bar{m}_{i} ., \ldots, x_{c r}-m_{c r}+\bar{m}_{c}\right.$. $)$. The function $f$ is strictly increasing, continuous and translatable since $\hat{f}$ is strictly increasing, continuous and translatable.

Next, we show that $f$ is symmetric. Let $X$ and $X^{\prime}$ be income distributions such that the vector $\left(x_{11}^{\prime}-m_{11}+\bar{m}_{1} ., \ldots, x_{i k}^{\prime}-m_{i k}+\bar{m}_{i} ., \ldots, x_{c r}^{\prime}-m_{c r}+\bar{m}_{c}\right.$. $)$ is obtained from the vector $\left(x_{11}-m_{11}+\bar{m}_{1 .}, \ldots, x_{i k}-m_{i k}+\bar{m}_{i}, \ldots, x_{c r}-m_{c r}+\bar{m}_{c}.\right)$ by a switch of two components. First, assume the switch is between the components corresponding to individuals $(i, k)$ and $(j, k)$. Note that $m_{i k}-\bar{m}_{i} .=m_{j k}-\bar{m}_{j}$. by additive separability of $M$. Because the same value $\left(m_{i k}-\bar{m}_{i} .=m_{j k}-\bar{m}_{j}\right.$.) is subtracted from the incomes $x_{i k}$ and $x_{j k}$, the switch is equivalent to a switch of these incomes. Hence, $W(X)=W\left(X^{\prime}\right)$ by ex post symmetry. Second, assume the switch is between the components corresponding to individuals $(i, k)$ and $(i, l)$. This switch is equivalent to a switch of the subsidies $x_{i k}-m_{i k}$ and $x_{i l}-m_{i l}$. Hence, we have $W(X)=W\left(X^{\prime}\right)$ by liberal symmetry. Third, assume the switch is between the components corresponding to individuals $(i, k)$ and $(j, l)$. Let $Y$ be the income distribution such that $\left(y_{11}-m_{11}+\bar{m}_{1} ., \ldots, y_{i k}-m_{i k}+\bar{m}_{i}, \ldots, y_{c r}-m_{c r}+\bar{m}_{c}\right.$. $)$ is obtained from the vector $\left(x_{11}-m_{11}+\bar{m}_{1 .}, \ldots, x_{i k}-m_{i k}+\bar{m}_{i .}, \ldots, x_{c r}-m_{c r}+\bar{m}_{c}\right.$. $)$ by a switch of the components corresponding to individuals $(i, l)$ and $(j, l)$. Using the same reasoning as above, by ex post symmetry, we have $W(X)=W(Y)$. Let $Y^{\prime}$ be the income distribution such that $\left(y_{11}^{\prime}-m_{11}+\bar{m}_{1 .}, \ldots, y_{i k}^{\prime}-m_{i k}+\bar{m}_{i .}, \ldots, y_{c r}^{\prime}-m_{c r}+\bar{m}_{c .}\right)$ is obtained from the vector $\left(y_{11}-m_{11}+\bar{m}_{1 .}, \ldots, y_{i k}-m_{i k}+\bar{m}_{i .}, \ldots, y_{c r}-m_{c r}+\bar{m}_{c}\right.$. $)$ by a switch of the components corresponding to individuals $(i, k)$ and $(i, l)$. Using the same reasoning as above, by liberal symmetry, we have $W(Y)=W\left(Y^{\prime}\right)$. The vector $\left(x_{11}^{\prime}-\right.$ $\left.m_{11}+\bar{m}_{1 .}, \ldots, x_{i k}^{\prime}-m_{i k}+\bar{m}_{i .}, \ldots, x_{c r}^{\prime}-m_{c r}+\bar{m}_{c}.\right)$ is obtained from the vector $\left(y_{11}^{\prime}-\right.$ $m_{11}+\bar{m}_{1} ., \ldots, y_{i k}^{\prime}-m_{i k}+\bar{m}_{i} ., \ldots, y_{c r}^{\prime}-m_{c r}+\bar{m}_{c}$.) by a switch of the components corresponding to individuals $(i, l)$ and $(j, l)$. Using the same reasoning as above, by ex post symmetry, we have $W\left(X^{\prime}\right)=W\left(Y^{\prime}\right)$. Thus, we obtain $W(X)=W\left(X^{\prime}\right)$.

Finally, we show that $f$ is strictly Schur-concave. Since $f$ is symmetric, it suffices to show that $f$ is Pigou-Dalton consistent. Let $X$ and $X^{\prime}$ be income distributions such that the vector $\left(x_{11}^{\prime}-m_{11}+\bar{m}_{1 .}, \ldots, x_{i k}^{\prime}-m_{i k}+\bar{m}_{i} ., \ldots, x_{c r}^{\prime}-m_{c r}+\bar{m}_{c .}\right)$ is obtained from the vector $\left(x_{11}-m_{11}+\bar{m}_{1} ., \ldots, x_{i k}-m_{i k}+\bar{m}_{i} ., \ldots, x_{c r}-m_{c r}+\bar{m}_{c}.\right)$ by a single progressive transfer. First, assume the transfer is from the component corresponding to individual $(i, k)$ to the component corresponding to individual $(j, k)$. Because the same value is subtracted from the incomes $x_{i k}$ and $x_{j k}$, this transfer is equivalent to a progressive transfer of income between $(i, k)$ and $(j, k)$. Hence, $W(X)>W\left(X^{\prime}\right)$ by ex post Pigou-Dalton. Second, assume the transfer is from the component corresponding to individual $(i, k)$ to the component corresponding to individual $(i, l)$. This transfer is equivalent to a progressive transfer from the subsidy $x_{i k}-m_{i k}$ to the subsidy $x_{i l}-m_{i l}$. Hence, we have $W(X)>W\left(X^{\prime}\right)$ by liberal Pigou-Dalton. Third, assume the transfer is from the component corresponding to individual $(i, k)$ to the component corresponding to $(j, l)$. Let $Y$ be the income distribution 
such that $\left(y_{11}-m_{11}+\bar{m}_{1 .}, \ldots, y_{i k}-m_{i k}+\bar{m}_{i .}, \ldots, y_{c r}-m_{c r}+\bar{m}_{c .}\right)$ is obtained from the vector $\left(x_{11}-m_{11}+\bar{m}_{1}, \ldots, x_{i k}-m_{i k}+\bar{m}_{i} ., \ldots, x_{c r}-m_{c r}+\bar{m}_{c}\right.$. $)$ by a switch of the components corresponding to individuals $(i, l)$ and $(j, l)$. Using the same reasoning as above, by ex post symmetry, we have $W(X)=W(Y)$. Let $Y^{\prime}$ be the income distribution such that $\left(y_{11}^{\prime}-m_{11}+\bar{m}_{1 .}, \ldots, y_{i k}^{\prime}-m_{i k}+\bar{m}_{i}, \ldots, y_{c r}^{\prime}-m_{c r}+\bar{m}_{c}.\right)$ is obtained from the vector $\left(y_{11}-m_{11}+\bar{m}_{1} ., \ldots, y_{i k}-m_{i k}+\bar{m}_{i} ., \ldots, y_{c r}-m_{c r}+\bar{m}_{c}\right.$. $)$ by a transfer from the component corresponding to $(i, k)$ to the component corresponding to $(i, l)$. Using the same reasoning as above, by liberal Pigou-Dalton, we have $W\left(Y^{\prime}\right)>W(Y)$. The vector $\left(x_{11}^{\prime}-m_{11}+\bar{m}_{1 .}, \ldots, x_{i k}^{\prime}-m_{i k}+\bar{m}_{i .}, \ldots, x_{c r}^{\prime}-m_{c r}+\bar{m}_{c .}\right)$ is obtained from the vector $\left(y_{11}^{\prime}-m_{11}+\bar{m}_{1 .}, \ldots, y_{i k}^{\prime}-m_{i k}+\bar{m}_{i} ., \ldots, y_{c r}^{\prime}-m_{c r}+\bar{m}_{c}.\right)$ by a switch of the components corresponding to individuals $(i, l)$ and $(j, l)$. Using the same reasoning as above, by ex post symmetry, we have $W\left(X^{\prime}\right)=W\left(Y^{\prime}\right)$. Thus, we obtain $W\left(X^{\prime}\right)>W(X)$.

Proof of Proposition 2 Let $W$ be a social welfare function that satisfies liberal Pigou-Dalton and ex ante symmetry.

Assume to the contrary that market incomes are not additively separable. That is, there exist $i$ and $j$ in $C$ and $k$ and $l$ in $R$ such that $m_{i k}-m_{i l} \neq m_{j k}-m_{j l}$. Let $X$ be an income distribution such that there exist positive real numbers $\alpha$ and $\beta$ such that $x_{i} .=m_{i} \cdot+\alpha 1_{r}$ and $x_{j} .=m_{j} .+\beta 1_{r}$ with $\min _{k \in R} x_{i k}>\max _{k \in R} x_{j k}$. Let $X^{\prime}$ be the income distribution obtained from $X$ by switching the $i$ th and the $j$ th rows of $X$. Let $X^{\prime \prime}$ be the income distribution obtained from $X^{\prime}$ by an income transfer of an amount $\epsilon$ between individuals $(i, k)$ and $(i, l)$ that corresponds to a progressive transfer in their subsidies. Moreover, let $\epsilon<\min _{k \in R} x_{i k}-\max _{k \in R} x_{j k}$, which implies that $\min _{k \in R} x_{j k}^{\prime \prime}>\max _{k \in R} x_{i k}^{\prime \prime}$. Let $X^{\prime \prime \prime}$ be the income distribution obtained from $X^{\prime \prime}$ by switching the $i$ th and the $j$ th rows of $X^{\prime \prime}$.

We have $W(X)=W\left(X^{\prime}\right)$ by ex ante symmetry, $W\left(X^{\prime}\right)<W\left(X^{\prime \prime}\right)$ by liberal PigouDalton and $W\left(X^{\prime \prime}\right)=W\left(X^{\prime \prime \prime}\right)$ by ex ante symmetry. Hence, $W(X)<W\left(X^{\prime \prime \prime}\right)$. However, we have $W(X)>W\left(X^{\prime \prime \prime}\right)$ by liberal Pigou-Dalton. We have a contradiction.

Proof of Theorem 4 It is easy to verify that the specified social welfare function satisfies the axioms. We focus on the reverse implication.

Let $W$ be a social welfare function that satisfies the axioms. By monotonicity, continuity and ex post aggregation, there exist a strictly increasing and continuous function $h: \mathbb{R}^{r} \rightarrow \mathbb{R}$ and strictly increasing and continuous functions $f_{1}, f_{2}, \ldots, f_{r}: \mathbb{R}^{c} \rightarrow \mathbb{R}$ such that, for each $X$ in $\mathbb{R}^{c \times r}$, we have $W(X)=h\left(f_{1}\left(x_{\cdot 1}\right), f_{2}\left(x_{\cdot 2}\right), \ldots, f_{r}\left(x_{\cdot r}\right)\right)$. Using uniform utilitarian symmetry, we can define strictly increasing and continuous functions $\hat{g}: \mathbb{R}^{r} \rightarrow \mathbb{R}$ and $\hat{f}: \mathbb{R}^{c} \rightarrow \mathbb{R}$ such that, for each $X$ in $\mathbb{R}^{c \times r}$, we have $W(X)=$ $\hat{g}\left(\hat{f}\left(x_{\cdot 1}\right), \hat{f}\left(x_{\cdot 2}\right), \ldots, \hat{f}\left(x_{\cdot r}\right)\right)$.

Translation invariance implies that, for all $x$ and $x^{\prime}$ in $\mathbb{R}^{c}$ and each real number $\lambda$, we have $\hat{f}(x) \geq \hat{f}\left(x^{\prime}\right)$ if and only if $\hat{f}\left(x+\lambda 1_{c}\right) \geq \hat{f}\left(x^{\prime}+\lambda 1_{c}\right)$, i.e., $\hat{f}$ is a translatable function. Hence, there exist a strictly increasing and continuous function $\psi: \mathbb{R} \rightarrow \mathbb{R}$ and a unit-translatable function $f: \mathbb{R}^{c} \rightarrow \mathbb{R}$ such that $\hat{f}=\psi \circ f$. Define the strictly increasing and continuous function $g: \mathbb{R}^{c} \rightarrow \mathbb{R}$ such that, for each $\left(t_{1}, t_{2}, \ldots, t_{r}\right)$ in $\mathbb{R}^{r}$, we have $g\left(t_{1}, t_{2}, \ldots, t_{r}\right)=\hat{g}\left(\psi\left(t_{1}\right), \psi\left(t_{2}\right), \ldots, \psi\left(t_{r}\right)\right)$. It follows that, for each $X$ in $\mathbb{R}^{c \times r}$, we have $W(X)=g\left(f\left(x_{\cdot 1}\right), f\left(x_{\bullet_{2}}\right), \ldots, f\left(x_{\cdot r}\right)\right)$. Note that by translation invariance, $g$ is translatable. The function $f$ is strictly increasing and continuous because $g$ and $\hat{f}$ are strictly increasing and continuous. Moreover, $f$ is symmetric by ex post symmetry, Pigou-Dalton consistent by ex post Pigou-Dalton, and hence strictly Schur-concave.

Next we show that $W$ is a strictly increasing function of $\frac{1}{r} \sum_{k \in R} f\left(x_{x_{k}}\right)$. Let $X$ and $X^{\prime}$ be income distributions in $\mathbb{R}^{c \times r}$. Let $Y$ and $Y^{\prime}$ in $\mathbb{R}^{c \times r}$ be income distributions 
such that $y_{i k}=f\left(x_{\cdot k}\right)$ and $y_{i k}^{\prime}=f\left(x_{\cdot k}^{\prime}\right)$ for each $(i, k)$ in $C \times R$. We have $W(Y)=g\left(f\left(f\left(x_{\cdot 1}\right) 1_{c}\right), f\left(f\left(x_{\cdot 2}\right) 1_{c}\right), \ldots, f\left(f\left(x_{\bullet r}\right) 1_{c}\right)\right)$. Since $f$ is unit-translatable, we have $W(Y)=g\left(f\left(x_{\cdot 1}\right)+\alpha, f\left(x_{\cdot 2}\right)+\alpha, \ldots, f\left(x_{\cdot r}\right)+\alpha\right)$ and $W\left(Y^{\prime}\right)=g\left(f\left(x_{\cdot 1}^{\prime}\right)+\right.$ $\left.\alpha, f\left(x_{.2}^{\prime}\right)+\alpha, \ldots, f\left(x_{. r}^{\prime}\right)+\alpha\right)$, where $\alpha=f\left(0 \times 1_{c}\right)$. Next, let $Z$ and $Z^{\prime}$ be income distributions such that $z_{i k}=\frac{1}{r} \sum_{k \in R} f\left(x_{\cdot k}\right)$ and $z_{i k}^{\prime}=\frac{1}{r} \sum_{k \in R} f\left(x_{\cdot k}^{\prime}\right)$ for each $(i, k)$ in $C \times R$. By monotonicity, $W(Z) \geq W\left(Z^{\prime}\right)$ if and only if $\frac{1}{r} \sum_{k \in R} f\left(x_{\cdot k}\right) \geq \frac{1}{r} \sum_{k \in R} f\left(x_{\cdot k}^{\prime}\right)$. By uniform utilitarian Pigou-Dalton, $W(Y)=W(Z)$ and $W\left(Y^{\prime}\right)=W\left(Z^{\prime}\right)$. Hence, $W(Y) \geq W\left(Y^{\prime}\right)$ if and only if $\frac{1}{r} \sum_{k \in R} f\left(x_{\cdot k}\right) \geq \frac{1}{r} \sum_{k \in R} f\left(x_{\cdot k}^{\prime}\right)$. We have already established that $W(X) \geq W\left(X^{\prime}\right)$ if and only if $W(Y) \geq W\left(Y^{\prime}\right)$. Therefore, $W(X) \geq W\left(X^{\prime}\right)$ if and only if $\frac{1}{r} \sum_{k \in R} f\left(x_{\cdot k}\right) \geq \frac{1}{r} \sum_{k \in R} f\left(x_{\cdot k}^{\prime}\right)$. It follows that there exists a strictly increasing and continuous function $F: \mathbb{R} \rightarrow \mathbb{R}$ such that $W(X)=F\left(\frac{1}{r} \sum_{k \in R} f\left(x_{\cdot k}\right)\right)$ for each $X$ in $\mathbb{R}^{c \times r}$.

Proof of Theorem 5 It is easy to verify that the specified social welfare function satisfies the axioms. We focus on the reverse implication.

Let $W$ be a social welfare function that satisfies the axioms. First, we show that, for each $X$ and $X^{\prime}$ in $\mathbb{R}^{c \times r}$, if $\sum_{k \in R} x_{i k}=\sum_{k \in R} x_{i k}^{\prime}$ for each $i$ in $C$, then we have $W(X)=W\left(X^{\prime}\right)$. Let $Y$ be the income distribution obtained from $X$ such that for each individual $(i, k)$, we have $y_{i k}=\bar{x}_{i}$. and let $Y^{\prime}$ be the income distribution obtained from $X^{\prime}$ such that for each individual $(i, k)$, we have $y_{i k}^{\prime}=\bar{x}_{i}^{\prime}$. By utilitarian reward, we have $W(X)=W(Y)$ and $W\left(X^{\prime}\right)=W\left(Y^{\prime}\right)$. By construction, $Y=Y^{\prime}$ and hence $W(X)=W\left(X^{\prime}\right)$.

Furthermore, if $\sum_{k \in R} x_{i k} \geq \sum_{k \in R} x_{i k}^{\prime}$ for each $i$ in $C$ with at least one inequality holding strictly, then we have $W(X)>W\left(X^{\prime}\right)$. This follows using monotonicity and the reasoning above. It follows that there exists a strictly increasing function $f: \mathbb{R}^{c} \rightarrow \mathbb{R}$ such that, for each $X$ in $\mathbb{R}^{c \times r}$, we have $W(X)=f\left(\bar{x}_{1 .}, \bar{x}_{2 .}, \ldots, \bar{x}_{c}\right.$. $)$. The function $f$ is continuous by continuity, symmetric by ex ante symmetry and translatable by translation invariance.

Next, we show that $f$ is strictly Schur-concave. Let $X$ and $X^{\prime}$ in $\mathbb{R}^{c \times r}$ be such that the vector $\left(\bar{x}_{1 .}, \bar{x}_{2 .}, \ldots, \bar{x}_{c}\right)$ is obtained from the vector $\left(\bar{x}_{1 .}^{\prime}, \bar{x}_{2 .}^{\prime}, \ldots, \bar{x}_{c}^{\prime}\right)$ by a progressive transfer. Let $Y$ be an income distribution such that $y_{i} .=\bar{x}_{i} .1_{r}$ for each $i$ in $C$, and let $Y^{\prime}$ be an income distribution such that $y_{i}^{\prime}=\bar{x}_{i}^{\prime} 1_{r}$ for each $i$ in $C$. Utilitarian reward implies that $W(X)=W(Y)$ and $W\left(X^{\prime}\right)=W\left(Y^{\prime}\right)$. Ex ante Pigou-Dalton implies that $W(Y)>W\left(Y^{\prime}\right)$. Hence, we have $W(X)>W\left(X^{\prime}\right)$. That is, $f$ is a Pigou-Dalton consistent function. Since it is also symmetric, by Lemma $2, f$ is Schur-concave.

Proof of Proposition 4 Let $W$ be a social welfare function that satisfies monotonicity, continuity and translation invariance.

(i) Let $W$ satisfy, in addition, ex post compensation and liberal reward, and let market incomes in $M$ be additively separable (Theorem 1). Let $X$ be an income distribution in $\mathbb{R}^{c \times r}$. First, to find $\Xi(X)$, we look for the optimal income distribution $Y$ such that $W(Y)=W(X)$. Since $Y$ is optimal, we have $y_{i k}=m_{i k}-\bar{m}_{i} .+\bar{Y}$ for each $(i, k)$ in $C \times R$ by Proposition 3(i). By Theorem $1, W(Y)=f(\bar{Y}, \ldots, \bar{Y})$ and $W(X)=f\left(x_{11}-m_{11}+\bar{m}_{1} ., \ldots, x_{i k}-m_{i k}+\right.$ $\left.\bar{m}_{i} ., \ldots, x_{c r}-m_{c r}+\bar{m}_{c}.\right)$. Since $W(Y)=W(X)$, we have $f(\bar{Y}, \ldots, \bar{Y})=f\left(x_{11}-m_{11}+\right.$ $\left.\bar{m}_{1 .}, \ldots, x_{i k}-m_{i k}+\bar{m}_{i} ., \ldots, x_{c r}-m_{c r}+\bar{m}_{c}.\right)$. Hence, $\bar{Y}=\xi\left(x_{11}-m_{11}+\bar{m}_{1 .}, \ldots, x_{i k}-\right.$ $m_{i k}+\bar{m}_{i}, \ldots, x_{c r}-m_{c r}+\bar{m}_{c}$.), where $\xi$ is the equally distributed equivalent income associated with $f$. Since $\bar{Y}=\Xi(X)$, we obtain $I(X)=\bar{X}-\xi\left(x_{11}-m_{11}+\bar{m}_{1 .}, \ldots, x_{i k}-\right.$ $m_{i k}+\bar{m}_{i .}, \ldots, x_{c r}-m_{c r}+\bar{m}_{c}$. $)$, i.e., $I(X)=J\left(x_{11}-m_{11}+\bar{m}_{1 .}, \ldots, x_{i k}-m_{i k}+\right.$ $\bar{m}_{i}, \ldots, x_{c r}-m_{c r}+\bar{m}_{c}$. $)$, where $J$ is the KAS inequality measure associated with $f$ in Eq. 1. The proofs of (ii) and (iii) are similar and are therefore omitted. 
(iv) Let $W$ satisfy, in addition, ex post compensation and uniform utilitarian reward (Theorem 4). Let $X$ be an income distribution in $\mathbb{R}^{c \times r}$. Let $Y$ be an income distribution in $\mathbb{R}^{c \times r}$ such that $y_{i k}=\alpha_{k} 1_{c}$ and $f\left(\alpha_{k} 1_{c}\right)=f\left(x_{\cdot k}\right)$ for each $k$ in $R$. By Proposition 3(iv), $Y$ is optimal and by Theorem $4, W(X)=W(Y)$. For each $k$ in $R$, we have $\alpha_{k}=\xi\left(x_{\cdot k}\right)$, where $\xi$ is the equally distributed equivalent income associated with $f$. Using $\Xi(X)=\bar{Y}=\sum_{k \in R} \alpha_{k} / r=\sum_{k \in R} \xi\left(x_{\cdot k}\right) / r$ and $X=\sum_{k \in R} \bar{x}_{x \cdot k} / r$, we obtain $I(X)=\bar{X}-\Xi(X)=\sum_{k \in R}\left[\bar{x}_{\cdot k}-\xi\left(\bar{x}_{\cdot k}\right)\right] / r$, i.e., $I(X)=\sum_{k \in R} J(x \cdot k) / r$, where $J$ is the KAS inequality measure associated with $f$ in Eq. 4.

(v) Let $W$ satisfy, in addition, ex ante compensation and utilitarian reward (Theorem 5). Let $X$ be an income distribution in $\mathbb{R}^{c \times r}$. Again, we look for an optimal distribution $Y$ such that $W(Y)=W(X)$. Since $Y$ is optimal, we have $\bar{y}_{i}$. $=\bar{y}_{j}$. for all circumstance groups $i$ and $j$ in $C$ by Proposition 3(v). By Theorem 5, $W(Y)=f(\bar{Y}, \ldots, \bar{Y})$ and $W(X)=f\left(\bar{x}_{1 .}, \bar{x}_{2 .}, \ldots, \bar{x}_{c}.\right)$. Since $W(Y)=W(X)$, we have $f(\bar{Y}, \ldots, \bar{Y})=$ $f\left(\bar{x}_{1}, \bar{x}_{2 .}, \ldots, \bar{x}_{c}\right.$. $)$. Hence, $\bar{Y}=\xi\left(\bar{x}_{1}, \bar{x}_{2 .}, \ldots, \bar{x}_{c}\right)$, where $\xi$ is the equally distributed equivalent income associated with $f$. We obtain that $I(X)=\bar{X}-\xi\left(\bar{x}_{1 .}, \bar{x}_{2 .}, \ldots, \bar{x}_{c}\right.$.), i.e., $I(X)=J\left(\bar{x}_{1}, \bar{x}_{2} ., \ldots, \bar{x}_{c}.\right)$, where $J$ is the KAS inequality measure associated with $f$ in Eq. 5.

Acknowledgments We thank Francisco Ferreira, Marc Fleurbaey, Jean-Jacques Herings, Erwin Ooghe, Dirk Van de gaer and audiences in Aix-en-Provence (Aix-Marseille Université), Bari (Fifth Meeting of the Society for the Study of Economic Inequality (ECINEQ)), Leuven (KU Leuven), Louvain-la-Neuve (Université catholique de Louvain) and Lund (Thirteenth Meeting of the Society for Social Choice and Welfare) for useful comments.

Open Access This article is licensed under a Creative Commons Attribution 4.0 International License, which permits use, sharing, adaptation, distribution and reproduction in any medium or format, as long as you give appropriate credit to the original author(s) and the source, provide a link to the Creative Commons licence, and indicate if changes were made. The images or other third party material in this article are included in the article's Creative Commons licence, unless indicated otherwise in a credit line to the material. If material is not included in the article's Creative Commons licence and your intended use is not permitted by statutory regulation or exceeds the permitted use, you will need to obtain permission directly from the copyright holder. To view a copy of this licence, visit http://creativecommons.org/licenses/by/4.0/.

\section{References}

Aaberge, R., Mogstad, M., Peragine, V.: Measuring long-term inequality of opportunity. J. Publ. Econ. 95(3), 193-204 (2011)

Almås, I., Cappelen, A.W., Lind, J.T., Sørensen, E.Ø., Tungodden, B.: Measuring unfair (in)equality. J. Public Econ. 95(7), 488-499 (2011)

Álvarez, A.S., Menéndez, A.J.L.: Assessing changes over time in inequality of opportunity: The case of Spain. Soc. Indic. Res. 139(3), 989-1014 (2018)

Andreoli, F., Havnes, T., Lefranc, A.: Robust inequality of opportunity comparisons: Theory and application to early childhood policy evaluation. Rev. Econ. Stat. 101(2), 355-369 (2019)

Arneson, R.J.: Equality and equal opportunity for welfare. Philos. Stud. 56(1), 77-93 (1989)

Arneson, R.J.: Liberalism, distributive subjectivism, and equal opportunity for welfare. Phil. Publ. Affairs 19(2), 158-194 (1990)

Assaad, R., Krafft, C., Roemer, J., Salehi-Isfahani, D.: Inequality of opportunity in wages and consumption in Egypt. Rev. Income Wealth 64(S1), 26-54 (2018)

Atkinson, A.B.: On the measurement of inequality. J. Econ. Theory 2(3), 244-263 (1970)

Belhaj Hassine, N.: Inequality of opportunity in Egypt. World Bank Econ. Rev. 26(2), 265-295 (2012)

Blackorby, C., Bossert, W., Donaldson, D.: Income Inequality Measurement: The Normative Approach. In: Silber, J. (ed.) Handbook of Income Inequality Measurement, pp. 133-157. Kluwer, Dordrecht (1999) 
Bosmans, K., Dormans, B., Öztürk, Z.E.: Failure to compensate or failure to reward? A decomposition of inequality of opportunity (2020)

Bosmans, K., Öztürk, Z.E.: Laissez-faire versus Pareto. Adam Smith Business School Discussion Paper 2015-21. University of Glasgow (2015)

Bosmans, K., Öztürk, Z.E.: Equality and responsibility: Ex ante and ex post redistribution mechanisms (2021)

Bossert, W.: Redistribution mechanisms based on individual characteristics. Math. Soc. Sci. 29(1), 1-17 (1995)

Bossert, W., Fleurbaey, M.: Redistribution and compensation. Soc. Choice Welf. 13(3), 343-355 (1996)

Bossert, W., Fleurbaey, M., Van de gaer, D.: Responsibility, talent, and compensation: a second-best analysis. Rev. Econ. Des. 4(1), 35-55 (1999)

Bourguignon, F., Ferreira, F.H.G., Menéndez, M.: Inequality of opportunity in Brazil. Rev. Income Wealth 53(4), 585-618 (2007)

Brunori, P.: The perception of inequality of opportunity in Europe. Rev. Income Wealth 63(3), 464-491 (2017)

Brunori, P., Palmisano, F., Peragine, V.: Inequality of opportunity in sub-Saharan Africa. Appl. Econ. 51(60), 6428-6458 (2019)

Cappelen, A.W., Tungodden, B.: Fairness and the proportionality principle. Soc. Choice Welf. 49(3-4), 709719 (2017)

Checchi, D., Peragine, V.: Inequality of opportunity in Italy. J. Econ. Inequal. 8(4), 429-450 (2010)

Checchi, D., Peragine, V., Serlenga, L.: Fair and unfair income inequalities in Europe. IZA Discussion Paper 5025 (2015)

Cogneau, D., Mesplé-Somps, S.: Inequality of Opportunity for Income in Five Countries of Africa. In: Bishop, J., Zheng, B. (eds.) Inequality and Opportunity: Papers from the Second ECINEQ Society Meeting (Research on Economic Inequality, vol. 16), pp. 99-128. Emerald Group Publishing Limited (2008)

Cohen, G.A.: On the currency of egalitarian justice. Ethics 99(4), 906-944 (1989)

Cowell, F.A.: Measures of distributional change: an axiomatic approach. Rev. Econ. Stud. 52(1), 135-151 (1985)

Cowell, F.A.: Measurement of Inequality. In: Atkinson, A.B., Bourguignon, F. (eds.) Handbook of Income Distribution, vol. 1, pp. 87-166. Elsevier, Amsterdam (2000)

Dasgupta, P., Sen, A., Starrett, D.: Notes on the measurement of inequality. J. Econ. Theory 6(2), 180-187 (1973)

Devooght, K.: To each the same and to each his own: a proposal to measure responsibility-sensitive income inequality. Economica 75(298), 280-295 (2008)

Dworkin, R.: What is equality? Part 1: Equality of welfare. Phil. Publ. Affairs 10(3), 185-246 (1981a)

Dworkin, R.: What is equality? Part 2: Equality of resources. Phil. Publ. Affairs 10(4), 283-345 (1981b)

Ebert, U.: Measurement of inequality: an attempt at unification and generalization. Soc. Choice Welf. 5(2-3), 147-169 (1988)

Ferreira, F.H.G., Gignoux, J.: The measurement of inequality of opportunity: Theory and an application to Latin America. Rev. Income Wealth 57(4), 622-657 (2011)

Ferreira, F.H.G., Gignoux, J., Aran, M.: Measuring inequality of opportunity with imperfect data: The case of Turkey. J. Econ. Inequal. 9(4), 651-680 (2011)

Ferreira, F.H.G., Peragine, V.: Individual Responsibility and Equality of Opportunity. In: Adler, M.D., Fleurbaey, M. (eds.) The Oxford Handbook of Well-Being and Public Policy, pp. 746-784. Oxford University Press, Oxford (2016)

Fleurbaey, M.: On fair compensation. Theory Decis. 36(3), 277-307 (1994)

Fleurbaey, M.: Fairness, Responsibility and Welfare. Oxford University Press, Oxford (2008)

Fleurbaey, M., Maniquet, F.: Compensation and Responsibility. In: Arrow, K.J., Sen, A., Suzumura, K. (eds.) Handbook of Social Choice and Welfare, vol. 2, pp. 507-604. Elsevier, Amsterdam (2011)

Fleurbaey, M., Peragine, V.: Ex ante versus ex post equality of opportunity. Economica 80(317), 118-130 (2013)

Fleurbaey, M., Peragine, V., Ramos, X.: Ex post inequality of opportunity comparisons. Soc. Choice Welf. 49(3-4), 577-603 (2017)

Fleurbaey, M., Schokkaert, E.: Unfair inequalities in health and health care. J. Health Econ. 28(1), 73-90 (2009)

Fleurbaey, M., Schokkaert, E.: Equity in Health and Health Care. In: Pauly, M.V., McGuire, T.G., Barros, P.P. (eds.) Handbook of Health Economics, vol. 2, pp. 1003-92. Elsevier, Amsterdam (2012)

Hufe, P., Peichl, A., Roemer, J., Ungerer, M.: Inequality of income acquisition: The role of childhood circumstances. Soc. Choice Welf. 49(3-4), 499-544 (2017) 
Kolm, S.-C.: The Optimal Production of Social Justice. In: Julius. M., Henri, G. (eds.) Public Economics: An Analysis of Public Production and Consumption and Their Relations to the Private Sectors, pp. 145-200. Macmillan, London (1969)

Kolm, S.-C.: Unequal inequalities I. J. Econ. Theory 12(3), 416-442 (1976)

Kranich, L.: Equitable opportunities: An axiomatic approach. J. Econ. Theory 71(1), 131-147 (1996)

Lefranc, A., Pistolesi, N., Trannoy, A.: Inequality of opportunities vs. inequality of outcomes: Are Western societies all alike? Rev. Income Wealth 54(4), 513-546 (2008)

Lefranc, A., Pistolesi, N., Trannoy, A.: Equality of opportunity and luck: Definitions and testable conditions, with an application to income in France. J. Public Econ. 93(11-12), 1189-1207 (2009)

Mack, E., Gaus, G.F.: Classical Liberalism and Libertarianism: The Liberty Tradition. In: Gaus, G.F., Kukathas, C. (eds.) Handbook of Political Theory. Sage, London (2004)

Mahler, D.G., Ramos, X.: Equality of opportunity in four measures of well-being. Rev. Income Wealth 65(S1), S228-S255 (2019)

Marrero, G.A., Rodríguez, J.G.: Inequality of opportunity and growth. J. Dev. Econ. 104, 107-122 (2013)

Moyes, P.: A new concept of Lorenz domination. Econ. Lett. 23(2), 203-207 (1987)

Moyes, P.: Stochastic Dominance and the Lorenz Curve. In: Silber, J. (ed.) Handbook of Income Inequality Measurement, pp. 199-222. Kluwer, Dordrecht (1999)

Ok, E.A.: On opportunity inequality measurement. J. Econ. Theory 77(2), 300-329 (1997)

Ok, E.A., Kranich, L.: The measurement of opportunity inequality: A cardinality-based approach. Soc. Choice Welf. 15(2), 263-287 (1998)

Olkin, I., Marshall, A.W.: Inequalities: Theory of Majorization and Its Applications. Academic Press, New York (1979)

Ooghe, E., Schokkaert, E., Van de gaer, D.: Equality of opportunity versus equality of opportunity sets. Soc. Choice Welf. 28(2), 209-230 (2007)

Peichl, A., Ungerer, M.: Accounting for the spouse when measuring inequality of opportunity. Soc. Choice Welf. 47(3), 607-631 (2016)

Peragine, V.: Measuring and implementing equality of opportunity for income. Soc. Choice Welfare 22(1), 187-210 (2004a)

Peragine, V.: Ranking income distributions according to equality of opportunity. J. Econ. Inequal. 2(1), 11-30 (2004b)

Pignataro, G.: Equality of opportunity: Policy and measurement paradigms. J. Econ. Surv. 26(5), 800-834 (2012)

Piraino, P.: Intergenerational earnings mobility and equality of opportunity in South Africa. World Dev. 67, 396-405 (2015)

Ramos, X., Van de gaer, D.: Approaches to inequality of opportunity: Principles, measures and evidence. J. Econ. Surv. 30(5), 855-883 (2016)

Ramos, X., Van de gaer, D.: Is inequality of opportunity robust to the measurement approach? Rev. Income Wealth 67(1), 18-36 (2021)

Rawls, J.: A Theory of Justice. Harvard University Press, Cambridge (1971)

Roemer, J.E.: A pragmatic theory of responsibility for the egalitarian planner. Phil. Publ. Affairs 22(2), 146-166 (1993)

Roemer, J.E.: On several approaches to equality of opportunity. Econ. Philos. 28(2), 165-200 (2012)

Roemer, J.E., Trannoy, A.: Equality of Opportunity. In: Atkinson, A.B., Bourguignon, F. (eds.) Handbook of Income Distribution, vol. 2A, pp. 217-300. Elsevier, Amsterdam (2015)

Roemer, J.E., Trannoy, A.: Equality of opportunity: Theory and measurement. J. Econ. Lit. 54(4), 1288-1332 (2016)

Sen, A.: On Economic Inequality. Clarendon Press, Oxford (1973)

Shorrocks, A.F.: Ranking income distributions. Economica 50(197), 3-17 (1983)

Singh, A.: Inequality of opportunity in earnings and consumption expenditure: The case of Indian men. Rev. Income Wealth 58(1), 79-106 (2012)

Van de gaer, D.: Equality of Opportunity and Investment in Human Capital. Katholieke Universiteit Leuven, Faculteit der Economische en Toegepaste Economische Wetenschappen (1993)

Weymark, J.A.: Generalized Gini indices of equality of opportunity. J. Econ. Inequal. 1(1), 5-24 (2003)

Publisher's note Springer Nature remains neutral with regard to jurisdictional claims in published maps and institutional affiliations. 